Ilkka Nissilä, Tommi Noponen, Jenni Heino, Timo Kajava, and Toivo Katila. 2005. Diffuse optical imaging. In: James C. Lin (editor). Advances in Electromagnetic Fields in Living Systems. New York, USA: Springer Science+Business Media, volume 4, chapter 3, pages 77-129.

(C) 2005 Springer Science+Business Media

Reprinted with kind permission from Springer Science+Business Media. 
Chapter 3

\title{
Diffuse Optical Imaging
}

\author{
Ilkka Nissilä, Tommi Noponen, Jenni Heino, Timo Kajava, \\ and Toivo Katila
}

\begin{abstract}
Diffuse optical imaging is a functional medical imaging modality which takes advantage of the relatively low attenuation of near-infrared light to probe the internal optical properties of tissue. The optical properties are affected by parameters related to physiology such as the concentrations of oxy- and deoxyhemoglobin. Instrumentation that is used for optical imaging is generally able to measure changes in the attenuation of light at several wavelengths, and in the case of time- and frequency-domain instrumentation, the time-of-flight of the photons in tissue.

Light propagation in tissue is generally dominated by scattering. Models for photon transport in tissue are generally based on either stochastic approaches or approximations derived from the radiative transfer equation. If a numerical forward model which describes the physical situation with sufficient accuracy exists, inversion methods may be used to determine the internal optical properties based on boundary measurements.

Optical imaging has applications in, e.g., functional brain imaging, breast cancer detection, and muscle imaging. It has the important advantages of transportable instrumentation, relatively high tolerance for external electromagnetic interference, non-invasiveness, and applicability for neonatal studies. The methods are not yet in clinical use, and further research is needed to improve the reliability of the experimental techniques, and the accuracy of the models used.
\end{abstract}

Ilkka Nissilä, Tommi Noponen, Jenni Heino, Timo Kajava, and Toivo Katila $\quad$ Laboratory of Biomedical Engineering, Helsinki University of Technology, P.O. Box 2200, 02015 HUT, Finland.

Advances in Electromagnetic Fields in Living Systems, Volume 4, edited by James C. Lin, Springer Science+Business Media, New York, 2005. 
In this chapter, we describe the interaction between light and tissue, light propagation models and inversion methods used in optical tomography, and instrumentation and experimental techniques used in optical imaging. The main applications of optical imaging are described in detail, with results from recent literature.

\section{INTRODUCTION}

Near-infrared light penetrates relatively easily into tissue due to the existence of the socalled optical window of tissue in the wavelength range from 650 to $950 \mathrm{~nm}$. A significant absorber in this wavelength range is hemoglobin. Jöbsis (1977) demonstrated that it is possible to monitor changes in the blood volume and tissue oxygenation in the brain noninvasively by measuring the attenuation of near-infrared light at several wavelengths. Hoshi and Tamura (1993) showed that the increased blood flow and oxygenation due to brain activation can also be detected using near-infrared spectroscopy (NIRS).

The nature of light propagation in tissue is complicated by the strong scattering of most tissue types. In order to develop an imaging modality based on the measurement of the characteristics of the light that has traveled through tissue, a model which accurately describes scattering and absorption in tissue should be used. Most researchers have used models based on the radiative transfer equation (RTE) or approximations derived from it. Most applications of near-infrared measurements to the human body require the measurement of the light that has traveled through several centimeters of tissue. In this case, light propagation can be considered to be diffuse.

Optical imaging using diffuse near-infrared light is primarily a functional imaging modality. The goal is to obtain quantitative information about changes in the optical properties inside the tissue using boundary measurements. The light is guided into tissue using source optodes distributed on the surface of the tissue, and detected using optodes typically placed in between the source optodes (Fig. 1a). In addition to the intensity of light, datatypes which describe the distribution of photon travel times through the tissue can be measured using time-resolved techniques.

In order to resolve the optical properties inside the tissue, model-based reconstruction techniques are needed. The sensitivity of optical measurements to changes in the internal

a)

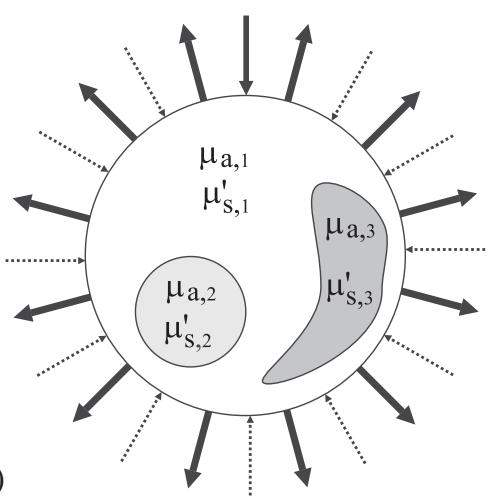

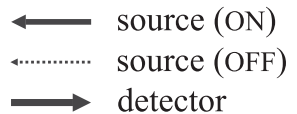

detector b)

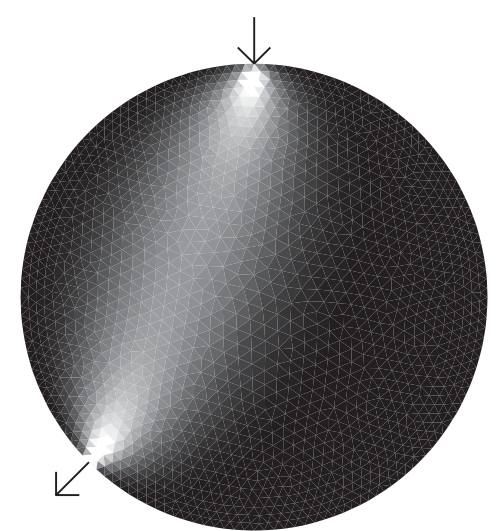

Figure 1. a) Principle of optical tomography. b) The sensitivity of the amplitude to changes in the absorption coefficient. 
optical properties can be calculated based on the light propagation model. An illustration of the result of sensititivity analysis in the two-dimensional case is shown in Fig. 1b. The forward model and sensitivity analysis can be used in an iterative manner to search a distribution of optical properties which explains the boundary measurements. The inverse problem is ill-posed in the sense that small errors in the measurements may lead to large errors in the reconstructed images.

In addition to the anatomical contrast due to different optical properties of tissue types, diffuse optical imaging can be used to image changes in perfusion and oxygen consumption. This is especially important since the spatial resolution of diffuse optical imaging is not comparable with anatomical medical imaging modalities such as X-ray imaging or magnetic resonance imaging $(\mathrm{MRI})$.

Instruments for diffuse optical imaging can be classified into three groups: time-domain systems which measure the full temporal point spread function of tissue to a pulse of light (Chance et al., 1988; Delpy et al., 1988, Eda et al., 1999; Schmidt et al., 2000), frequencydomain systems which measure the amplitude and phase shift of the detected light with respect to an intensity-modulated source light (Lakowicz and Berndt, 1990; Chance et al., 1998b; McBride et al., 2001; Nissilä et al., 2002a), and continuous wave systems, which measure the DC intensity of detected light (Schmitz et al., 2002). The primary advantage of time- and frequency-domain techniques is that they may provide enough information for the separation of features in scattering and absorption.

Advantages of optical imaging as a functional imaging modality include noninvasiveness, insensitivity to external electromagnetic interference, portability of the instrumentation, and suitability for neonatal studies. Problems in the local perfusion of the head of premature neonates may be revealed using optical tomography (Hebden et al., 2002). Optical tomography shows promise as a non-ionizing method for the detection of breast cancer (Franceschini et al., 1997; Pogue et al., 2001; Deghani et al., 2002). The oxidative metabolism of muscle tissue can also be studied with diffuse optical imaging.

In section 2, we discuss the basic mechanisms of light-tissue interaction especially in the near infared, as well as some physiological background. Section 3 reviews the mathematical models used for light transport in tissue-like highly scattering media. Techniques for image reconstruction are also discussed. In section 4, the different types of instruments used for diffuse optical imaging are described. In section 5, we review some of the most important applications of diffuse optical imaging and present some of the newest results from the literature.

\section{TISSUE OPTICS AND PHYSIOLOGY}

Proper interpretation of the data of diffuse optical imaging requires the understanding of optical properties and physiology of the underlying tissue. These issues are briefly presented in this section.

\subsection{Photon and Single-Particle Interactions}

The propagation of electromagnetic waves in a medium is traditionally modeled using the Maxwell equations. In diffuse optical imaging, such a complete description is not necessary because most tissues are strongly scattering in the near-infrared (NIR) region 
so that the wave properties such as polarization and interference of light can be ignored after the light has traversed a few millimeters of tissue. Light-tissue interactions can also change the structure of tissue or even destroy it. Such interaction mechanisms are utilized, e.g., in phototherapy and laser surgery. In optical imaging, the intensity levels are so low $\left(\mathrm{mW} / \mathrm{mm}^{2}\right)$ that these destructive processes do not occur. Furthermore, inelastic scattering has a negligible effect on the propagation of NIR light so that the contribution of processes such as fluorescence, phosphorescence, and Raman scattering can be neglected. Therefore, absorption and elastic scattering are the dominant photon-tissue interaction mechanisms in the NIR region.

\subsubsection{Absorption}

Absorption in tissue is often due to a chromophore, which is a special group in a molecule. The absorbed electromagnetic energy is usually converted into thermal energy. The absorption properties of chromophores depend on environmental factors, such as the type of host molecule, $\mathrm{pH}$, and temperature.

2.1.1a. Basics of Absorption. Absorption properties of a material can be characterized by the specific extinction coefficient $\alpha$. The product $c \alpha$, where $c$ is the concentration of the absorbing compound, is proportional to its absorption coefficient. It represents the probability that a photon is absorbed within a unit length in the medium and is typically given in $\mathrm{mm}^{-1}$. In the case of a single absorbing compound and no scattering, the intensity of light $I$ as a function of pathlength $x$ is obtained from the Beer-Lambert law as

$$
\frac{I(x)}{I_{0}}=e^{-\mu_{a} x},
$$

where $I_{0}$ is the intensity of incident light.

The concentrations of absorbers in tissue can vary both spatially and temporally and the extinction coefficient is generally wavelength dependent. Because several chromophores contribute to the total absorption, the absorption coefficient is expressed as

$$
\mu_{a}(\mathbf{r}, t, \lambda)=\ln (10) \sum_{i} c_{i}(\mathbf{r}, t) \alpha_{i}(\lambda)
$$

The specific extinction coefficient is usually defined using base 10 logarithms and $\mu_{a}$ using natural logarithms.

2.1.1b. Absorption in Tissue. Approximately $75 \%$ of the human body is water (Woodard and White, 1986). Due to its abundance, water is a considerable absorber of NIR light. Fortunately, the absorption of water is relatively low in the wavelength range of 200-950 nm (see Fig. 2).

Lipids have absorption properties similar to those of water. 1-80\% of tissue is lipids, depending on the location within the body (Woodard and White, 1986). Because of their lower relative concentration, lipids do not typically contribute significantly to the total light attenuation. However, this does not apply to adipose tissue of the breast for example, which is mostly composed of lipids.

In surface tissues, the absorption of light, and especially of ultraviolet light, is relatively strong. In the NIR region, melanin is the dominant absorbing chromophore of the epidermis 


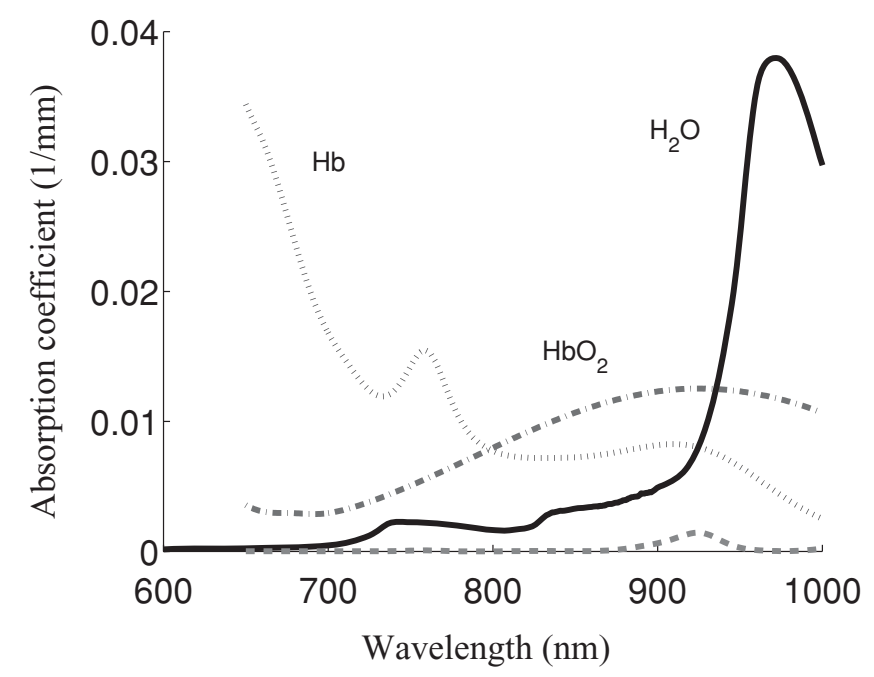

Figure 2. Scaled absorption coefficients of water (solid), $\mathrm{Hb}$ (dotted), $\mathrm{HbO}_{2}$ (dashdot), and lipids (dashed) in the NIR region (Matcher et al., 1994; Cope, 1991; Quaresima et al., 1998).

layer of skin. However, the epidermal layer of the body is rather thin so that its contribution to the total attenuation of NIR light is relatively low.

Especially in brain and muscle studies, the local concentrations of water and lipids, as well as the optical properties of some surface tissues and bones, whose absorption of NIR light is relatively low, are often considered constant. These substances with several weaker absorbers comprise the group of fixed background absorbers. However, the local variations in the concentration of hemoglobin (an oxygen carrier molecule in the erythrocyte) can be relatively large. Its absorption properties depend on the oxygenation state, because the absorption spectra of oxygenated $\left(\mathrm{HbO}_{2}\right)$ and deoxygenated $(\mathrm{Hb})$ hemoglobin are notably different in the NIR region (see Fig. 2). These properties are fundamental to functional NIR imaging of tissue. The absorption coefficient of hemoglobin is also rather low above $650 \mathrm{~nm}$. This fact together with the absorption properties of water limit the suitable wavelength region to $650-950 \mathrm{~nm}$ when thick objects $(\sim \mathrm{cm})$ are studied. In this region, non-invasive studies of local concentration changes of $\mathrm{Hb}$ and $\mathrm{HbO}_{2}$ are possible provided that the true mean pathlength of photons in the tissue is known and at least two wavelengths are used (Jöbsis, 1977).

Cytochrome oxidase is an enzyme in the mitochondrial membrane taking part in the cellular respiratory chain. Its absorption properties depend on its redox state. The oxidized and reduced forms of the enzyme have different absorption spectra and its total concentration stays relatively constant in tissue. It might be therefore possible to use a single additional NIR wavelength to estimate the redox state changes of cytochrome oxidase, provided that the difference spectrum of the specific extinction coefficients of different forms of the enzyme is known (Cope, 1991).

\subsubsection{Scattering}

The dominant light-tissue interaction in the NIR region is elastic scattering, where the energy of a photon stays constant but its direction changes. Scattering theories provide 
many useful methods for modeling light propagation in tissue (Ishimaru, 1978; Bohren and Huffman, 1983). The Rayleigh scattering theory is frequently used when the size of the scattering particle of any shape is much less than the wavelength of light. The Mie scattering theory can be applied to particles of any size as long as they have a spherical shape. Also, more advanced theories are used in tissue optics (see Sect. 3).

2.1.2a. Basics of Scattering. The scattering properties of a single particle are expressed by a scattering cross section $\sigma$. The total scattering coefficient $\mu_{s}$ of a medium which is composed of different scatterers is given by

$$
\mu_{s}=\sum_{i} \sigma_{i} \frac{N_{i}}{V}
$$

where $N_{i}$ is the number of scatterers in a volume $V$. The scattering coefficient is a quantity which describes the probability per unit length that a photon is scattered and is expressed usually in $\mathrm{mm}^{-1}$. The reciprocal $1 / \mu_{s}$ characterizes the mean free path between consecutive scattering events. In the case of single scattering events, an exponential attenuation law similar to Eq. (1) can be defined for scattering. However in a larger volume of tissue, multiple-scattering effects have to be taken into account, which requires more advanced modeling (see Sect. 3).

Scattering in tissue is usually anisotropic. A measure of the directional dependency of scattering is given by the normalized scattering phase function $f_{\hat{\mathrm{s}}}\left(\hat{\mathrm{s}}, \hat{\mathrm{s}}^{\prime}\right)$. This is a probability density function of $\hat{\mathrm{s}}$ for scattering from an incident direction $\hat{\mathrm{s}}^{\prime}$ into a new direction $\hat{s}$. If the scattering is independent on the absolute direction of the incident light, $f_{\hat{\mathrm{s}}}\left(\hat{\mathrm{s}}, \hat{\mathrm{s}}^{\prime}\right)$ depends only on the angle $\theta$ between the incident and scattered directions and $f_{\hat{\mathrm{s}}}\left(\hat{\mathrm{s}}, \hat{\mathrm{s}}^{\prime}\right) d \hat{\mathrm{s}}=$ $f_{\cos \theta}(\cos \theta) d \cos \theta$. This is a commonly used approximation for many tissue types.

The mean cosine of the scattering phase function can be written as

$$
g=2 \pi \int_{-1}^{1} \cos \theta f_{\cos \theta}(\cos \theta) d \cos \theta
$$

This parameter is zero for isotropic scattering. When $g \rightarrow 1$, scattering vanishes, and when $g \rightarrow-1$, scattering becomes completely backward directed. The $g$ parameter is often called the anisotropy factor.

If scattering is anisotropic, the effective scattering properties of a medium can be described with an effective (or reduced or transport) scattering coefficient. It is defined as

$$
\mu_{s}^{\prime}=(1-g) \mu_{s} .
$$

In addition to the absorption coefficient, the effective scattering coefficient is a material parameter often estimated in diffuse optical tomography.

2.1.2b. Scattering in Tissue. Scattering takes place at each boundary where the refractive index $n$ changes. ${ }^{\dagger}$ In tissue, such boundaries exist, e.g., between the intra- and extracellular fluid and between the cytoplasm and the interior fluid of organelles. On a macroscopic scale the differences in refractive indices are relatively small and their values are about 1.4 in most tissues (Bolin et al., 1989). The significant differences can mainly be found at air-skin interfaces. Therefore $n$ is often considered to be constant throughout the medium.

\footnotetext{
$\dagger$ The refractive index determines, e.g., the speed of light in a medium.
} 
Scattering in tissue is slightly wavelength dependent, because it is mostly due to particles whose dimensions are close to the incident wavelength. Also the shape and density of particles as well as environmental variables such as temperature typically influence scattering (Laufer et al., 1998). The spatial origin of $\mu_{s}^{\prime}$ is not easy to specify. Cellular membranes compose a large fraction of the total volume of tissue and they contain scattering boundaries so that they should contribute extensively to scattering (Cope, 1991). Mitochondria and other cellular organelles have appropriate sizes for NIR scattering and they are distributed all over the body as well (Beauvoit and Chance, 1998; Mourant et al., 1998). Scattering due to red blood cells is probably rather insignificant because their solid content in the body is rather low (about 2\%) (Cope, 1991).

The macroscopic structure of some tissues may produce a preferred direction for light propagation. In muscle tissue, light tends to propagate along muscle fibers because of structural anisotropy (Marquez et al., 1998). The same may apply to the white matter of the brain due to the axon bundles.

\subsubsection{Determination of Concentration Changes in Tissue}

In near-infrared spectroscopy (NIRS), total attenuation of light is often modeled using the modified Beer-Lambert law,

$$
A=\log _{10} \frac{I_{0}}{I}=\alpha \cdot c \cdot d \cdot D P F+G,
$$

where $d$ is the geometrical distance between the observation points and the parameter $G$ represents attenuation due to scattering. $D P F$ is the so-called differential pathlength factor, which characterizes the average pathlength of photons in the tissue, given by $d \cdot D P F$. The documented values of $D P F$ for human tissue typically vary between 3.5 and 6.5 (Elwell, 1995).

For simplicity, the attenuation due to scattering and background absorption is often considered constant. Then, the concentration changes of a chromophore can be estimated from Eq. (6). When a medium contains $N$ chromophores of interest and the number of measurement wavelengths is $M(N \leq M)$, these changes are given by

$$
\Delta \mathbf{c}=\left(\alpha^{T} \alpha\right)^{-1} \alpha^{T} \frac{\Delta \boldsymbol{A}}{d \cdot D P F},
$$

where $\Delta \mathbf{c}$ is a $\mathrm{N} \times 1$ vector and $\Delta \mathbf{A}$ is a $\mathbf{M} \times 1$ vector containing the changes in attenuation. The matrix $\alpha(\mathrm{M} \times \mathrm{N})$ contains the specific extinction coefficients for each chromophore at each wavelength.

Equation (7) is widely used in NIRS. In addition to the assumption of fixed scattering and background absorption, the DPF is often taken as a constant. In practice, however, it depends on the wavelength, the measurement geometry, and the $\mu_{a}$ and $\mu_{s}^{\prime}$ of the medium.

\subsection{Optical Properties of Tissues}

A priori information of the optical properties of tissue can be used to study the feasibility of optical imaging, such as the expected contrast between benign and malignant tissue. It 
can also be applied, e.g., to select appropriate measurement and modeling techniques and reconstruction algorithms, and to verify results.

\subsubsection{Measurement and Modeling Techniques}

Techniques for the determination of optical properties can be divided into direct and indirect methods (Wilson et al., 1987). Direct methods produce the material parameters directly from measurement data applying rather simple modeling techniques such as exponential laws. Therefore, direct methods are limited to cases where a single or only a few scattering events occur, making possible only the studies of thin tissue samples (often $\leq 100 \mu \mathrm{m})$. Direct methods can be used to measure, e.g., the total attenuation coefficient $\left(\mu_{t}=\mu_{a}+\mu_{s}\right)$ of tissue and values of the scattering phase function.

Indirect methods usually require advanced light propagation models (Cheong et al., 1990). The integrating-sphere technique is a classic indirect measurement setup (Pickering et al., 1993). The simplest model for such measurements is based on the Kubelka-Munk theory. However, it requires a nearly isotropic diffuse flux inside the sample and may produce significant errors if the model and the measurement setup are inconsistent (Cheong et al., 1990; van der Zee, 1992). Therefore, other modeling techniques such as the adding-doubling method and Monte Carlo simulation are often applied to integrating sphere measurements (Prahl et al., 1993; van der Zee, 1992). The integrating sphere technique is suitable for in vitro samples only and edge effects in the measurement setup limit the sample thickness (typically $\leq 1 \mathrm{~mm}$ ).

In vivo techniques allow measurements through thicker tissue samples (normally $\geq$ $1 \mathrm{~cm}$ ), in which case $\mu_{a}$ and $\mu_{s}^{\prime}$ are appropriate material parameters. In practice, such measurements are often preferred. Organs are, however, more difficult to access and the estimation of the measured volume is not straightforward. In vivo studies also require advanced measurement techniques and light propagation models (see Sects. 3 and 4).

\subsubsection{Parameters of Some Tissue Types}

There are some compilations of optical properties of different tissue types (Cheong et al., 1990; van der Zee, 1992; Tuchin, 2000). In Table 1, we have listed the optical properties of relevant tissues from recent studies. The data shows that practically all tissues are strongly scattering $\left(\mu_{\mathrm{a}} \ll \mu_{\mathrm{s}}^{\prime}\right)$. Only in cerebrospinal fluid (CSF) $\mu_{a}$ is considerably larger than $\mu_{s}^{\prime}$. In most cases tissue is also strongly forward scattering (typically $0.70<g<0.995$ ). ${ }^{\ddagger}$

\subsection{Physiological and Structural Changes Measured in Diffuse Optical Imaging}

The functional imaging capability of diffuse optical imaging is mostly based on changes in the attenuation of NIR light due to the changes in oxidative metabolism in tissue. The

\footnotetext{
$\ddagger$ There is some variation in tissue properties reported by different authors. This may be due to differences in measurement setups, instrumentation, in vivo versus in vitro, modeling, preparation of tissue samples, and/or environmental factors such as temperature. The composition and heterogeneity of samples can also contribute to these variations.
} 
Table 1. Optical Properties of Some Tissues Studied Using Diffuse Optical Imaging. Some Values in the Table Have Been Calculated from the Available Data (+), Whereas Other Values Have Been Approximated from Graphs (*).

\begin{tabular}{|c|c|c|c|c|}
\hline Tissue & $\mu_{a}\left(\mathrm{~mm}^{-1}\right)$ & $\mu_{s}^{\prime}\left(\mathrm{mm}^{-1}\right)$ & $\mathrm{g}$ & $\lambda(\mathrm{nm})$ \\
\hline Muscle (abdominal) $^{+}$ & $0.0052-0.017$ & $0.64-0.95$ & - & $674-956^{1}$ \\
\hline Muscle (abdominal) ${ }^{+}$ & $0.025-0.17$ & $0.58-0.94$ & - & $618-950^{2}$ \\
\hline Muscle (back) $)^{+}$ & $0.0082-0.017$ & $0.55-1.2$ & - & $674-956^{1}$ \\
\hline Brain (grey matter) ${ }^{+}$ & $0.0090-0.026$ & $0.42-1.2$ & - & $674-956^{3}$ \\
\hline Brain $\left(\right.$ white matter) ${ }^{+}$ & $0.013-0.097$ & $0.68-1.5$ & - & $674-956^{3}$ \\
\hline Brain (grey matter)* & $0.02-0.07$ & $1.8-3.0$ & $0.95-0.97$ & $650-950^{4}$ \\
\hline Brain (white matter)* & $0.005-0.04$ & $7-10$ & $0.78-0.86$ & $650-950^{4}$ \\
\hline Brain (neonatal, grey mat)* & $0.02-0.05$ & $0.4-0.7$ & $0.98-0.99$ & $650-900^{4}$ \\
\hline Brain (neonatal, white mat)* & $0.03-0.05$ & $0.7-1.3$ & $0.97-0.98$ & $650-900^{4}$ \\
\hline Brain (CSF, approx. by pure water) ${ }^{+}$ & $0.0013-0.029$ & $\sim 0$ & - & $650-900^{5}$ \\
\hline Breast (adipose) ${ }^{+}$ & $\leq 0.035$ & $0.48-1.2$ & - & $749-836^{6}$ \\
\hline Breast (fibrous) ${ }^{+}$ & $\leq 0.032$ & $0.58-1.2$ & - & $749-836^{6}$ \\
\hline Breast (average)* & $0.002-0.008$ & $0.6-1.4$ & - & $750-830^{7}$ \\
\hline Blood* & $0.13-0.49$ & $2.5-4.0$ & $0.99-0.995$ & $665-960^{8,9}$ \\
\hline Bone (pig skull) ${ }^{+}$ & $0.022-0.052$ & $1.2-2.8$ & $0.91-0.96$ & $650-950^{10}$ \\
\hline Bone (human skull) ${ }^{+}$ & $0.02-0.07$ & $0.75-1.2$ & - & $674-956^{3}$ \\
\hline Skin (dermis, Caucasian) $)^{+}$ & $0.0053-0.049$ & $1.3-3.4$ & - & $618-950^{2}$ \\
\hline Skin (dermis, black) ${ }^{+}$ & $0.025-0.46$ & $1.1-5.5$ & - & $617-949^{2}$ \\
\hline Subdermal fat $^{+}$ & $0.0040-0.024$ & $0.8-1.7$ & - & $617-949^{2}$ \\
\hline
\end{tabular}

${ }^{1}$ Fishkin et al., 1997; ${ }^{2}$ Simpson et al., 1998; ${ }^{3}$ Bevilacqua et al., 1999; ${ }^{4}$ van der Zee et al., 1993; ${ }^{5}$ Matcher et al., 1994; ${ }^{6}$ Troy et al., 1996; ${ }^{7}$ Durduran et al., 2002; ${ }^{8}$ Cheong et al., 1990; ${ }^{9}$ Lovell et al., 1999a; ${ }^{10}$ Firbank et al., 1993

activity of neurons and muscle cells is controlled by electrical impulses (i.e., action potentials) along their cellular membranes. This activation is accompanied by increased nutrient and oxygen demand of cells, which tends to increase also the local blood flow.

In a typical brain-activation measurement using NIRS, $\left[\mathrm{HbO}_{2}\right]$ and [totHb] in tissue increase and [Hb] decreases (Fig. 3). ${ }^{\S} \mathrm{II} I t$ is generally accepted that during activation, blood flow and oxygen delivery in tissue increase more than oxygen consumption (Villringer and Dirnagl, 1995). An increase in blood velocity increases the $\left[\mathrm{HbO}_{2}\right]$ and decreases the $[\mathrm{Hb}]$. The blood volume can also increase regionally in capillaries. However, a local increase in blood volume would increase the $\left[\mathrm{HbO}_{2}\right]$ and [tot $\left.\mathrm{Hb}\right]$, but the $[\mathrm{Hb}]$ would stay practically constant. Changes in oxygen consumption can affect both $\left[\mathrm{HbO}_{2}\right]$ and $[\mathrm{Hb}]$. The increased oxygen consumption decreases the $\left[\mathrm{HbO}_{2}\right]$ and increases the $[\mathrm{Hb}]$ and vice versa. The probed region in NIRS measurements is typically so large $\left(>1 \mathrm{~cm}^{3}\right)$ that all the hemodynamic and oxygenation phenomena mentioned above can contribute to the measured signal.

The optical properties of cytochrome oxidase may enable an estimation of tissue oxygenation and cellular oxygen metabolism. The redox-active copper center $\mathrm{Cu}_{A}$ is mostly responsible for the absorption of NIR light in the cytochrome oxidase, but it is only indirectly responsible for the oxygen consumption. There is evidence that in addition to the cellular oxygen consumption, other processes can change the redox state of $\mathrm{Cu}_{A}$ (Cooper

\footnotetext{
$\S$ The brackets indicate the concentration of substance and the abbreviation totHb refers to the total hemoglobin.

II The drop in [Hb] also creates the so-called BOLD-contrast (blood oxygen level-dependent), which is mostly responsible for the signal changes in functional magnetic resonance imaging (fMRI).
} 


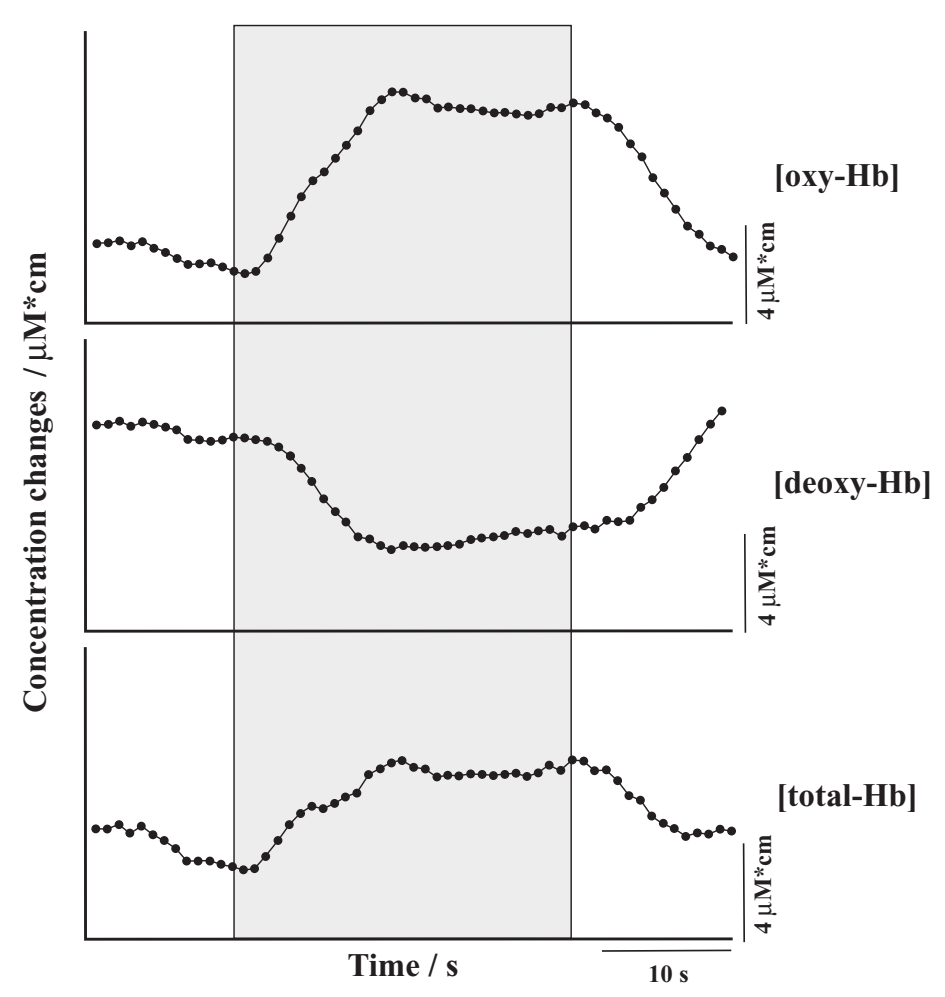

Figure 3. Typical NIRS response of a) $\left[\mathrm{HbO}_{2}\right]$, b) [Hb], and c) [totHb] to a visual randomly moving stimulus. The stimulus is on during the gray interval. NIRS signals were recorded above the right occipital cortex and the responses have been averaged over 8 subjects. Reproduced with permission from (Obrig and Villringer, 1997).

et al., 1994). The absorption spectrum of $\mathrm{HbO}_{2}$ also resembles the difference spectrum of cytochrome oxidase (Cope, 1991). These facts complicate the non-invasive determination of changes in the redox-state of cytochrome oxidase (Cooper and Springett, 1997).

Variations in the scattering properties of tissue can also be detected using optical techniques. The signals attributed to scattering changes can be categorized into fast and slow signals (Villringer and Chance, 1997). The published research on scattering changes is mostly from animal studies using invasive methods (Hill and Keynes, 1950; Frostig et al., 1990; Macvisar and Hochman, 1991). The fast scattering signals are related to neuronal activity and action potentials providing direct information on the electrical activity of tissue (Stepnoski et al., 1991). The time scale of these changes is typically milliseconds (Rector et al., 2001). The geometrical changes of cells and organelles in tissue have also been shown to produce changes in scattering (Poe et al., 1996). Geometrical changes can be quite fast (tens of milliseconds), but they have been typically used to explain slower scattering changes (Rector et al., 2001; Villringer and Chance, 1997). When absorption and scattering changes are simultaneously measured, one should be aware of the link between them. When scattering increases, photons tend to propagate longer distances in tissue, which increases the probability for absorption in the tissue and vice versa.

Vascular, metabolic, and neuronal systems produce periodic changes, which may show up in the optical signal. While at rest, the frequency of oscillations due to the heartbeat and the pulsation of arterial blood is approximately $1 \mathrm{~Hz}$. This fluctuation has been utilized to monitor the oxygen saturation of arterial blood (Franceschini et al., 1999). Features at 
respiratory frequencies $(0.2-0.3 \mathrm{~Hz})$ may also be seen in the optical signal (Elwell et al., 1996). Furthermore, the cognitive activation of the frontal lobe has been demonstrated to induce recurrent oscillations in the range of $0.5-5 \mathrm{~Hz}$ (Chance et al., 1993). Living tissue also produce low-frequency (LFO, around $0.1 \mathrm{~Hz}$ ) and very-low-frequency oscillations (VLFO, around $0.04 \mathrm{~Hz}$ ), which have been proposed to arise from vascular (such as vasomotion), metabolic and/or neuronal fluctuations (Obrig et al., 2000).

In addition to functional images, diffuse optical imaging can be used to image the optical structure of tissue at a low resolution (around $1 \mathrm{~cm}^{3}$ ). Structural imaging is based on the differences in the absorption and scattering coefficients between different tissue types (see Sect. 2.2.2). These differences can be utilized to detect, e.g., tumors, internal hemorrhages or hematomas (Zhang et al., 2000). Many studies of optical properties of malignant tissues have shown that the absorption and scattering coefficients may be larger than in benign tissues (Hargrave et al., 1996; Tromberg et al., 1997; Fantini et al., 1998).

\section{THEORY AND MODELING}

\subsection{Problem Statement}

The fundamental idea in optical tomography is that the internal distribution of $\mu_{s}$ and/or $\mu_{a}$ can be reconstructed based on a set of measurements of transmitted and/or reflected light between points on the boundary of an object. Reconstructing an image of optical parameters based on boundary measurements is an example of a so-called inverse problem. For solving an inverse problem, the solution to the respective forward problem is generally required.

Formally, the forward problem in optical imaging can be stated as: Given the distribution of sources $q$ on the boundary of the domain, and the distribution of the optical parameter values $p$ in the domain, find the resulting boundary measurement set $y$. The forward problem can be expressed with a non-linear forward operator:

$$
y=F(p ; q) .
$$

The inverse problem can be stated as: Given the boundary measurements y and the distribution of sources $q$ on the boundary, find the internal distribution of optical parameter values $p$. Using the non-linear operator, the inverse problem can be written formally as:

$$
p=F^{-1}(y ; q) .
$$

\subsection{Photon Transport Models}

\subsubsection{Radiative Transfer Equation}

Based on the particle interpretation, light can be studied as a stream of energetic particles, i.e., photons. If the photon density is interpreted as proportional to the scalar field for energy radiance $L$, various differential and integrodifferential equations can be established based on energy conservation. The most widely applied equation in the field 
of optical imaging is the Radiative Transfer Equation (RTE) (Case and Zweifel, 1967; Chandrasekhar, 1960)

$$
\begin{aligned}
\frac{1}{c} \frac{\partial L(\mathbf{r}, t, \hat{\mathbf{s}})}{\partial t}+\hat{\mathbf{s}} \cdot \nabla L(\mathbf{r}, t, \hat{\mathbf{s}} & +\left(\mu_{a}+\mu_{\mathrm{s}}\right) L(\mathbf{r}, t, \hat{\mathbf{s}}) \\
& =\mu_{s} \int_{(4 \pi)} f_{\hat{\mathbf{s}}}\left(\hat{\mathbf{s}}, \hat{\mathbf{s}}^{\prime}\right) L(\mathbf{r}, t, \hat{\mathbf{s}}) d \hat{\mathbf{s}}^{\prime}+q(\mathbf{r}, t, \hat{\mathbf{s}}),
\end{aligned}
$$

which describes the change in radiance $L(\mathbf{r}, t, \hat{\mathbf{s}})$ in time at position $\mathbf{r}$ and in direction $\hat{\mathbf{s}}$. Above, $c$ is the speed of light in the medium, $f_{\hat{\mathbf{s}}}\left(\hat{\mathbf{s}}, \hat{\mathbf{s}}^{\prime}\right)$ is the normalized scattering phase function and $q(\mathbf{r}, t, \hat{\mathbf{s}})$ is the spatial and angular distribution of the source.

The radiance $L$ is defined so that the energy transfer per unit time by photons in a unit solid angle $d \hat{\mathbf{s}}$ through an elemental area $d a$ with a unit normal $\hat{\mathbf{n}}$, at position $\mathbf{r}$, is given by

$$
L(\mathbf{r}, t, \hat{\mathbf{s}}) \hat{\mathbf{s}} \cdot \hat{\mathbf{n}} d a d \hat{\mathbf{s}}
$$

The exitance $\Gamma$ on surface, i.e., the energy transfer per unit time through a unit area with a normal $\hat{\mathbf{n}}$, is obtained from Eq. (11) by integrating over the solid angle:

$$
\Gamma(\mathbf{r}, t)=\int_{(4 \pi)} L(\mathbf{r}, t, \hat{\mathbf{s}}) \hat{\mathbf{s}} \cdot \hat{\mathbf{n}} d \hat{\mathbf{s}} .
$$

The units for the exitance are $\mathrm{Wm}^{-2}$ and for the radiance $\mathrm{Wm}^{-2} \mathrm{Sr}^{-1}$, where sr is the unit for the solid angle. The units for the source term $q$ in Eq. (10) are $\mathrm{Wm}^{-3} \mathrm{sr}^{-1}$.

For solving the inverse problem, a relatively fast and flexible solution to the forward problem is desirable. Although numerical solutions to the RTE have been presented, still normally simplified models derived from the RTE are used as a basis of image reconstruction.

\subsubsection{The Diffusion Approximation}

A standard approximation method for the RTE is the $P_{N}$ approximation (Case and Zweifel, 1967). The $P_{N}$ approximation consists of expanding the radiance $L(\mathbf{r}, t, \hat{\mathbf{s}})$ and the source term $q(\mathbf{r}, t, \hat{s})$ in Eq. (10) into spherical harmonics and taking terms only up to $N$ th order. Using the addition theorem of spherical harmonics, the scattering phase function can also be expanded into spherical harmonics (Arridge, 1999). Inserting these expansions in the RTE and some manipulation of the resulting expression yields in the general case $\mathrm{N}+1$ coupled first-order partial differential equations (PDEs).

The diffusion approximation (DA) (for a more complete presentation, see (Case and Zweifel, 1967; Ishimaru, 1978)) is obtained from the $P_{1}$ approximation by making some additional simplifying assumptions. It is the most commonly applied model for optical tomography though there has also been some work on higher order approximations such as the $P_{3}$ approximation to the RTE (Arridge, 1999; Boas, 1996; Jiang, 1999).

We now define the energy fluence rate

$$
\Phi(\mathbf{r}, t)=\int_{(4 \pi)} L(\mathbf{r}, t, \hat{\mathbf{s}}) d \hat{\mathbf{s}}
$$


and the energy current density

$$
\mathbf{J}(\mathbf{r}, t)=\int_{(4 \pi)} \hat{\mathbf{s}} L(\mathbf{r}, t, \hat{\mathbf{s}}) d \hat{\mathbf{s}}
$$

both in the units of $\mathrm{Wm}^{-2}$. In terms of the definitions (13) and (14), the $P_{1}$ approximation yields two coupled PDEs:

$$
\begin{gathered}
\frac{1}{c} \frac{\partial \Phi(\mathbf{r}, t)}{\partial t}+\nabla \cdot \mathbf{J}(\mathbf{r}, t)+\mu_{a} \Phi(\mathbf{r}, t)=q_{0}(\mathbf{r}, t), \\
\frac{1}{c} \frac{\partial \mathbf{J}(\mathbf{r}, t)}{\partial t}+\frac{1}{3} \nabla \Phi(\mathbf{r}, t)+\left(\mu_{a}+\mu_{s}(1-g)\right) \mathbf{J}(\mathbf{r}, t)=\mathbf{q}_{1}(\mathbf{r}, t),
\end{gathered}
$$

where $g$ is the mean cosine of the scattering angle. The $P_{1}$ approximation is quite good when $\mu_{s} /\left(\mu_{s}+\mu_{a}\right.$ is close to unity, i.e., $\mu_{a} \ll \mu_{s}$, the phase function is not too anisotropic, and the source-detector separation is large compared to the mean free scattering length $1 / \mu_{s}^{\prime}$ (Boas, 1996).

To derive the DA, we make the assumptions

$$
\begin{gathered}
\mathbf{q}_{1}(\mathbf{r}, t)=0, \\
\frac{1}{|\mathbf{J}|} \frac{\partial|\mathbf{J}|}{\partial t} \ll c\left(\mu_{a}+\mu_{s}^{\prime}\right) .
\end{gathered}
$$

The first assumption is justified if an isotropic source term is assumed. The second assumption effectively means that the left-hand-side term in Eq. (18) is taken to be zero. The implication of the second assumption is best seen in the frequency domain. The intensity of the source term is assumed to be sinusoidally modulated with angular frequency $\omega$ and the time dependence of the photon current $\mathbf{J}$ is thus of the form $e^{-i \omega t}$. The time derivatives can then be replaced by multiplying by $-i \omega$ and the second assumption (when $\mu_{s}^{\prime} \gg \mu_{a}$ ) thus essentially becomes $\omega /\left(c \mu_{s}^{\prime}\right) \ll 1$. This means that the scattering frequency must be much larger than the modulation frequency (Boas, 1996). This assumption is considered to be a good approximation for biological tissues, if the source frequencies are less than $\sim 1 \mathrm{GHz}$ (Haskell et al., 1994).

Substituting the assumptions (17) and (18) into Eq. (16) simplifies it to Fick's law,

$$
\mathbf{J}(\mathbf{r}, t)=-\frac{1}{3\left(\mu_{a}(\mathbf{r})+\mu_{s}^{\prime}(\mathbf{r})\right)} \nabla \Phi(\mathbf{r}, t)=-\kappa(\mathbf{r}) \nabla \Phi(\mathbf{r}, t),
$$

where $\kappa(\mathbf{r})$ is the diffusion coefficient,

$$
\kappa(\mathbf{r})=\frac{1}{3\left(\mu_{a}(\mathbf{r})+\mu_{s}^{\prime}(\mathbf{r})\right)} .
$$

Substituting Fick's law into Eq. (15) leads to the time-dependent diffusion equation (DE)

$$
\frac{1}{c} \frac{\partial \Phi(\mathbf{r}, t)}{\partial t}-\nabla \cdot \kappa(\mathbf{r}) \nabla \Phi(\mathbf{r}, t)+\mu_{a} \Phi(\mathbf{r}, t)=q_{0}(\mathbf{r}, t) .
$$

The exitance $\Gamma$ defined in Eq. (12) can now, using Eq. (14) and Fick's law, be written as

$$
\Gamma(\mathbf{r}, t)=-\kappa(\mathbf{r}) \hat{\mathbf{n}} \cdot \nabla \Phi(\mathbf{r}, t) .
$$


3.2.2a. Validity of the Diffusion Approximation. The DA is a simple and the most widely applied model for light propagation in optical imaging. It is valid for most tissue types, though there are some important exceptions. For example, it cannot be used to model light transport in clear regions (e.g., the CSF), where the assumption of strong scattering is violated. Also, in the proximity of light sources more rigorous models could yield more accurate results. In (Hielscher et al, 1997), diffusion and transport calculations in a head model were compared. It was found that most differences occured in void-like regions and in regions where the absorption was comparable to scattering. Comparisons of diffusion calculations with experiments or Monte Carlo simulations based on the RTE show, however, that qualitatively and also quantitatively correct predictions of boundary measurements can be obtained using diffusion models when the required assumptions for the DA are met (Arridge et al., 1993; Okada et al., 1996; Rinzema et al., 1998).

3.2.2b. Frequency Domain. A common experimental approach is to modulate the intensity of the light source with a radio-frequency signal. Consider a point source modulated with an angular frequency $\omega$ :

$$
q_{0}(\mathbf{r}, t)=\delta\left(\mathbf{r}-\mathbf{r}_{s}\right)\left(Q_{0}+S e^{-i \omega t}\right),
$$

where $Q_{0}$ is the average or the dc amplitude and $S$ the modulation amplitude of the source. In a strongly scattering medium an intensity-modulated point source gives rise to a macroscopic wave of photon density propagating spherically outward from the source in the medium. In the literature, these waves are often referred to as diffuse photon density waves (DPDW) (Boas, 1996; Fishkin and Gratton, 1993; O'Leary et al., 1992). One approach to optical imaging is based on studying the refraction and reflection of these waves. The DE in the frequency domain is obtained by taking the Fourier transform of the time-dependent DE (21):

$$
-\frac{i \omega}{c} \hat{\Phi}(\mathbf{r}, \omega)-\nabla \cdot \kappa(\mathbf{r}) \nabla \hat{\Phi}\left(\mathbf{r}, \omega+\mu_{a}(\mathbf{r}) \hat{\Phi}(\mathbf{r}, \omega)=\hat{q}_{0}(\mathbf{r}, \omega),\right.
$$

where $\hat{\Phi}(\mathbf{r}, \omega)$ is the complex fluence arising from the intensity modulated part $\hat{q_{0}}(\mathbf{r}, \omega)$ of the source term.

\subsubsection{Boundary and Source Conditions}

In order to achieve a better match between the light propagation model and the physical situation, the boundaries and sources must be carefully modelled (see, e.g., (Haskell et al., 1994; Schweiger et al., 1995)). A commonly applied approach in the literature is to use the zero or Dirichlet boundary condition (DBC), where the fluence is set equal to zero on the physical boundary:

$$
\Phi(\mathbf{r}, t)=0 \quad \forall \mathbf{r} \in \partial \Omega .
$$

The DBC, although not physically accurate, is mathematically quite simple.

Another, physically more correct boundary condition can be derived from the requirement that no photons come into the tissue through the surface (except at the source position) (Ishimaru, 1978):

$$
L(\mathbf{r}, t, \hat{\mathbf{s}})=0 \quad \forall \mathbf{r} \in \partial \Omega, \hat{\mathbf{s}} \cdot \hat{\mathbf{n}}<0
$$


This condition, however, cannot be applied to the diffusion equation directly. Instead, we assume that the total inward directed current is zero:

$$
\int_{\hat{\mathrm{s}} \cdot \hat{\mathbf{n}}<0} L(\mathbf{r}, t, \hat{\mathbf{s}})(\hat{\mathbf{s}} \cdot \hat{\mathbf{n}}) d \hat{\mathbf{s}}=0 \quad \forall \mathbf{r} \in \partial \Omega .
$$

Within the diffusion approximation, the boundary condition becomes

$$
\Phi(\mathbf{r}, t)+2 \kappa(\mathbf{r}) A \hat{\mathbf{n}} \cdot \nabla \Phi(\mathbf{r}, t)=0 \quad \forall \mathbf{r} \in \partial \Omega,
$$

where the term $A=(1+R) /(1-R)$ takes into account the differences in the refractive indices $n$ and $n^{\prime}$ of the scattering medium and the surrounding medium ( $R$ is the reflection coefficient on the boundary). In the literature, the above boundary condition is known as the zero partial flux or the Robin boundary condition (RBC).

The RBC represents the physical situation more accurately, whereas the DBC has the advantage of leading to a simpler model. A compromise between these two is the extrapolated boundary condition (EBC), where an extrapolated boundary is introduced at a certain distance $d_{\text {ext }}$ from the real physical boundary, and the DBC is then applied at this extrapolated boundary. Comparing with Monte Carlo simulations and experimental results, the RBC and EBC give much better agreement than the DBC (Okada et al., 1996; Schweiger et al., 1995).

In a measurement system for optical tomography, a laser light beam can be guided onto the surface of the tissue through an optical fiber. A common approach to model a collimated pencil beam incident on the surface is to represent it by an isotropic point source located at a depth $1 / \mu_{s}^{\prime}$ below the surface of the domain $\Omega$. This approximation produces rather accurate results at distances larger than one mean free path, i.e., $1 /\left(\mu_{s}^{\prime}+\mu_{a}\right)$, from the source, but breaks down close to the source. In the DE, the isotropic point source can be represented as a $\delta$-function source term.

Another approach is to treat the light source as a diffuse source on the surface of the domain. The source is represented as an inward-directed diffuse photon current over the illuminated area of the domain boundary $\partial \Omega$. The source can thus be incorporated directly into the boundary conditions.

\subsubsection{Measurables}

The measurable quantity on the boundary is the exitance $\Gamma$ (see Eq. (22)). Using the $\mathrm{RBC}(28) \Gamma$ can be written as

$$
\Gamma(\mathbf{r}, t)=\frac{1}{2 A} \Phi(\mathbf{r}, t) .
$$

Although the time-resolved flux intensity $\Gamma(t)$ could be used for reconstruction directly, commonly one or a few data types derived from $\Gamma(t)$ are used. Possible data types are numerous: the total integrated intensity, time-gated integrals (early light as the integral over the first few nanoseconds, or windowed light) or integral transform type measurements (Fourier, Mellin or Laplace transform and their combinations), which can be used to obtain, e.g., the mean-time or higher-order moments. If frequency-domain measurements are considered, $\Gamma(\omega)$ is usually calculated from frequency-domain DA. Calculation of $\Gamma(\omega)$ is often much simpler than obtaining $\Gamma(t)$. Following the presentation in (Schweiger and Arridge, 
1997; Schweiger and Arridge, 1999a), where the subject of data types is considered more profoundly, we give here the definitions of some of the common ones:

$$
\begin{aligned}
& \text { time - gated integrated intensity: } E=\int_{t_{1}}^{t_{2}} \Gamma(t) d t, \\
& \text { integrated intensity: } E=\int_{0}^{\infty} \Gamma(t) d t \\
& \text { nth temporal moment: }\left\langle t^{n}\right\rangle=E^{-1} \int_{0}^{\infty} t^{n} \Gamma(t) d t \\
& \text { th central moment: } \quad c_{n}=E^{-1} \int_{0}^{\infty}(t-\langle t\rangle)^{n} \Gamma(t) d t, \\
& \text { normalized Laplace transform: } L(s)=E^{-1} \int_{0}^{\infty} e^{-s t} \Gamma(t) d t \text {, } \\
& \text { Mellin - Laplace: } \quad M L_{n}(s)=E^{-1} \int_{0}^{\infty} t^{n} e^{-s t} \Gamma(t) d t, \\
& \text { AC intensity: }|\Gamma(\omega)|, \\
& \text { log intensity: } \quad \log \Gamma(\omega), \\
& \text { modulation depth: } \frac{\log \Gamma(\omega)}{E}, \\
& \text { phase: } \varphi=\arg \Gamma(\omega) .
\end{aligned}
$$

An important point is that all these data types except $E$ and $A C / \log$ intensity are normalized, so knowledge of the absolute magnitude is not required, which makes them more robust against variations in source power and effects of fiber-tissue coupling.

\subsection{Solving the Photon Transport}

\subsubsection{Stochastic Modeling}

The most widely applied stochastic technique for photon transport is the Monte Carlo (MC) method. In MC models (Wilson and Adam, 1983; Prahl et al., 1989), the propagation of an individual photon or a photon packet (to improve the efficiency of the method) is simulated through the medium. This is done by following the photon's path and modeling sequentially each event the photon undergoes during its travel in the medium.

MC techniques provide a conceptually simple way of modeling light propagation in an inhomogeneous medium with complex arbitrary geometry at the expense of increased computational complexity. The statistical error of MC results decreases with the inverse square root of the number of detected photons. In order to achieve acceptable statistics, the number of photons or photon packages that have to be launched is often high, resulting in computational times that hinder the use of MC models as a basis for iterative image reconstruction algorithms. However, MC methods have been widely used for investigating the behaviour of light or some parameters related to light propagation in complex inhomogeneous conditions (Okada et al., 1997), as well as for the validation of PDE-based light transport models (Schweiger et al., 1995). 


\subsubsection{Deterministic Approach}

In the deterministic approach, the RTE or PDEs derived from it are solved using either analytical or numerical methods.

Generally, numerical solutions to the RTE are computationally relatively expensive. Hence, most reconstruction algorithms rely on the diffusion approximation, though recently reconstructions based on the RTE have also been presented (Klose and Hielscher, 2002). Analytical solutions to the RTE are available only in some special cases, whereas for numerical solution, various computational techniques exist (Graaff et al., 1989; Kim and Ishimaru, 1998; Klose et al., 2002).

3.3.2a. Analytical Solutions to the DE. Analytical solutions to the diffusion equation are in general cases difficult to obtain. For some relatively simple geometries analytical solutions can be derived, but when dealing with arbitrary geometries or inhomogeneous media, one has to resort to numerical approaches.

For a point source in an infinite homogeneous medium, the solution of the DE is given by the Green function for infinite medium. For some other simple geometries, the solutions can be derived, e.g., using image sources and the infinite medium solution. Some geometries for which the DE has been solved in this manner include, e.g., semi-infinite half-space, an infinite slab or cylinder, and a two-dimensional circle or a three-dimensional sphere (Arridge et al., 1992; den Outer et al., 1993).

Some approaches for image reconstruction rely on studying the properties of the photon density field generated by an intensity-modulated light source (Boas et al., 2001; Boas et al., 1997; Chance et al., 1998; Ntziachristos et al., 1999). In a homogeneous medium, the frequency-domain diffusion equation (FDDE) (Eq. (24)) can be rewritten as the Helmholtz equation

$$
\left(\nabla^{2}+\kappa_{a c}^{2}\right) \hat{\Phi}\left(\mathbf{r}, \omega=-\frac{A}{\kappa} \delta\left(\mathbf{r}-\mathbf{r}_{s}\right)\right.
$$

with a complex wave number

$$
\kappa_{a c}^{2}=\frac{-c \mu_{a}+i \omega}{c \kappa}
$$

For an infinite homogeneous medium, the solution of Eq. (40) is an outgoing spherical wave:

$$
\hat{\Phi}(\mathbf{r}, \omega)=\frac{S}{4 \pi \kappa\left|\mathbf{r}-\mathbf{r}_{0}\right|} e^{i \kappa_{a c}\left|\mathbf{r}-\mathbf{r}_{0}\right|}
$$

where $S$ is the source modulation amplitude, $\kappa_{a c}=\sqrt{\left(-c \mu_{a}+i \omega\right) /(c \kappa)}$ the complex wave number, and $\left|\mathbf{r}-\mathbf{r}_{0}\right| r$ the distance from the source.

Analytical solutions for the propagation and scattering of the DPDWs have been studied in various relatively simple geometries and both in a homogeneous medium and in the presence of spherical perturbations (Feng et al., 1995; Fishkin and Gratton, 1993; O'Leary et al., 1992).

3.3.2b. Finite Difference Method. Finite Difference Method (FDM) is a classical numerical approach for solving PDEs. In the FDM, the solution area is discretized using a regular grid, and the derivatives are approximated by finite-difference approximations 
involving the values of the unknowns at adjacent grid points. Substituting the finitedifference approximations to the diffusion equation yields a discrete system equation.

FDM is conceptually simple and is often considered the easiest method to implement for regular domains. The system matrices have high degrees of symmetry, which makes the solution of the system equation easier. For complicated geometries, abrupt changes in material properties or complex boundary conditions, special modifications are, however, usually required. In optical tomography, the FDM is not the most common numerical method, but it has been used in inverse reconstruction schemes (Hielscher et al., 1999; Pogue et al., 1995).

3.3.2c. Finite Element Method. In the Finite Element Method (FEM), the discretization process begins from a variational formulation of the differential equation, where a set of so-called test functions is involved. The reduction to a finite-dimensional problem is made by replacing this (often infinite-dimensional) set by a set of functions depending only on a finite number of parameters and discretizing the solution area into a number of uniform or nonuniform finite elements. The result of this procedure can be reformulated to a system of equations with a sparse system matrix.

The FE formulation for the frequency-domain diffusion equation can be derived using the so-called Galerkin formulation. First, the FDDE is multiplied by a test function $\psi \in H^{1}(\Omega)$ (where $H^{1}(\Omega)$ is a predefined function space (Sobolev space)) and integrated over the solution domain $\Omega$. The resulting equation is called the weak formulation of the FDDE. In the Galerkin approximation, the solution to the weak formulation is found in an $N$-dimensional subspace $U(\Omega) \subset H^{1}(\Omega)$, where $N$ is finite. By defining a basis $U:\left\{\psi_{i}\right\}_{1}^{N}$ for the subspace $U(\Omega)$, the Galerkin approximation for $\hat{\Phi}$ can be expressed as

$$
\hat{\Phi}^{h}(\mathbf{r}, \omega)=\sum_{i=1}^{N} \hat{\Phi}_{i}(\omega) \psi_{i}(\mathbf{r}) .
$$

Assuming, e.g., a Robin-type boundary condition (Eq. (28)), the Galerkin formulation finally yields a finite set of equations:

$$
[\mathbf{A}+\mathbf{B}+\mathbf{C}] \hat{\Phi}=\hat{\mathbf{Q}}
$$

where

$$
\begin{aligned}
A_{j i} & =\int_{\Omega}\left[-\frac{i \omega}{c}+\mu_{a}(\mathbf{r})\right] \psi_{j}(\mathbf{r}) \psi_{i}(\mathbf{r}) d \Omega, \\
B_{j i} & =\int_{\Omega} \kappa(\mathbf{r}) \nabla \psi_{j}(\mathbf{r}) \cdot \nabla \psi_{i}(\mathbf{r}) d \Omega, \\
C_{j i} & =\int_{\partial \Omega} \frac{1}{2 A} \psi_{j}(\mathbf{r}) \psi_{i}(\mathbf{r}) d(\partial \Omega), \\
\hat{Q}_{j} & =\int_{\Omega} \hat{q}_{0}(\mathbf{r}, \omega) \psi_{j}(\mathbf{r}) d \Omega, \\
\hat{\Phi} & =\left[\hat{\Phi}_{1}(\omega), \hat{\Phi}_{2}(\omega), \ldots, \hat{\Phi}_{N}(\omega)\right]^{T} .
\end{aligned}
$$

In the time-dependent case, the FE formulation can be derived in a similar manner. The resulting FE matrix equation can be integrated with respect to time, the time variable discretized and the time derivatives approximated using the finite difference method. 
The versatility of the FEM makes complicated geometries, complex boundary conditions or a highly heterogeneous material relatively easy to handle. In optical tomography, FEM is a very commonly used method for solving the DE numerically.

\subsubsection{Hybrid Models}

Solving the DE with FEM is relatively fast compared to some other methods such as Monte Carlo simulation. However, the drawback of the DE is that it is not valid, e.g., close to the sources. With Monte Carlo methods, however, more realistic solutions near the sources can be obtained at the expense of computational time. The speed of the DE-based FEM and the accuracy of the Monte Carlo method can be combined in a hybrid model. Wang and Jacques (1993) simulated light propagation near the source with a Monte Carlo model and used the results to form a distributed source for the diffusion model.

\subsection{Inverse Problem}

\subsubsection{Uniqueness Issues}

The inverse problem of optical tomography is extremely ill-posed making the image reconstruction a complicated task. The question of the existence of a unique solution to the inverse problem has recently received certain attention. In theorical considerations, the existence of a unique parameter distribution consistent with boundary data depends on the measurement technique (steady-state, time-domain or frequency-domain) and boundary data types used, the optical parameters considered as unknown $\left(\mu_{a}, \mu_{s}\right.$, the refractive index $)$ and the underlying model for light propagation.

Image reconstruction using a transport model and some considerations on simultaneous reconstruction of absorption and scattering within the transport framework have been presented, e.g., by Dorn (1998, 2000, submitted). Within the diffusion model, a proof the nonuniqueness of recovering both the scattering and absorption coefficient distributions using steady-state measurements was given by Arridge and Lionheart (1998). The authors showed that for a steady-state measurement, there exists an infinite set of optical parameters that give rise to identical data. The discussion was then extended to frequency/time domain. In these cases the uniqueness could be proved in the limit of continuous measurements and source distributions provided that the refractive index was known. However, if the refractive index distribution is considered unknown the uniqueness disappears. The discussion was continued by Matcher (1999) using a more exact P1-approximation. Within this time-dependent model it could be argued that the solution for $\mu_{a}, \mu_{s}$ and refractive index distributions is almost certainly unique.

\subsubsection{Data Types for the Image Reconstruction}

The question of which data types to use for image reconstruction is closely related to the uniqueness issue. To our knowledge, formal results for different data types have not been derived. Some information on the usability of certain data types can, however, be obtained, e.g., by calculating the difference in the data caused by perturbations in the 
optical parameters. In (Arridge 1999; Schweiger and Arridge, 1997) the maps of the error norms $\varepsilon$ of the data were calculated in a homogeneous sphere with background parameters $\bar{\mu}_{a}$ and $\bar{\mu}_{s}^{\prime}$ and perturbed parameters $\mu_{a}$ and $\mu_{s}^{\prime}$ corresponding to a single perturbed area using:

$$
\varepsilon\left(\mu_{a}, \mu_{s}^{\prime}\right)=\sum_{i, j}\left(\frac{F_{i, j}\left(\bar{\mu}_{a}, \bar{\mu}_{s}^{\prime}\right)-F_{i, j}\left(\mu_{a}, \mu_{s}^{\prime}\right)}{\sigma_{i, j}}\right)^{2},
$$

where $F_{i, j}\left(\bar{\mu}_{a}, \bar{\mu}_{s}^{\prime}\right)$ and $F_{i, j}\left(\mu_{a}, \mu_{s}^{\prime}\right)$ are the homogeneous reference and the perturbed data, respectively, and $\sigma_{i, j}$ is the standard deviation for the measurement $(i, j)$. The error maps were plotted against $\left(\mu_{a}, \mu_{s}^{\prime}\right)$ axes. The deduction from the maps was that for a single measurement type simultaneous reconstruction of both $\mu_{a}$ and $\mu_{s}^{\prime}$ is ambiguous. However, combining measurement types in a suitable way can overcome the ambiguity between $\mu_{a}$ and $\mu_{s}^{\prime}$.

In order to employ a minimization scheme on Eq. (50), the standard deviations for the measurements need to be known. In experiments, the noise can be estimated based on the data. For simulated data, noise models have been established (Arridge et al., 1995; Schweiger and Arridge, 1997).

Yet another point to consider when choosing data types is the spatial sensitivity profile of the measurement. Different data types have slightly different spatial sensitivities, which can be accessed by calculating the sensitivity relations of the measurements with respect to parameter values.

\subsubsection{Sensitivity Relations}

The sensitivity relations between the measurement and the material parameters are utilized in many image reconstruction algorithms. For the forward model $y=F\left(\mathbf{r}_{m}, q, p\right)$ between the measurement $y$ at $\mathbf{r}_{m}$ and the parameter values $p$ and sources $q$, the sensitivity relation, often called as the Jacobian, can in general be defined as the rate of change of the measurement with $p$ :

$$
\mathcal{J}_{p}^{y}\left(\mathbf{r}_{m}, q, \mathbf{r}^{\prime}\right)=\lim _{\Delta p \rightarrow o} \frac{F\left(\mathbf{r}_{m}, q, p+\Delta p\left(\mathbf{r}^{\prime}\right)\right)-F\left(\mathbf{r}_{m}, q, p\right)}{\Delta p\left(\mathbf{r}^{\prime}\right)}
$$

The Jacobians for different data types can be obtained from the Jacobian for the exitance $\Gamma$, e.g., for $\log \Gamma$ and $\varphi=\arg \Gamma(\omega)$ the Jacobians become

$$
\begin{gathered}
\mathcal{J}_{p}^{\log \Gamma}=\operatorname{Re}\left[\frac{1}{\Gamma} \mathcal{J}_{p}^{\Gamma}\right] \text { and } \\
\mathcal{J}_{p}^{\varphi}=\operatorname{Im}\left[\frac{1}{\Gamma} \mathcal{J}_{p}^{\Gamma}\right],
\end{gathered}
$$

where $\mathcal{J}_{p}^{\Gamma}$ denotes the Jacobian for $\Gamma$.

Analytical forms of the Jacobian have been studied using the known analytical forms for the Green functions in some simple geometries (Arridge, 1995; Schotland et al., 1993). For general geometries and inhomogeneous conditions, Monte Carlo and finite element techniques have been used. If the model we are considering is discretized, the Jacobian can 
be written in a discrete form:

$$
\mathcal{J}_{p}^{y}=\left(\begin{array}{lll}
\frac{\partial_{y_{1}}}{\partial_{p_{1}}} & \cdots & \frac{\partial_{y_{1}}}{\partial_{p_{L}}} \\
\vdots & \ddots & \vdots \\
\frac{\partial_{Y M}}{\partial_{p_{1}}} & \cdots & \frac{\partial_{Y M}}{\partial_{p_{L}}}
\end{array}\right),
$$

where $y_{i}$, are the measurements at locations $1 \ldots M$ and $p_{j}$ are the parameter values in the discrete model. The computation of this Jacobian matrix is often burdensome and restricts the speed of image reconstruction algorithms.

There are several ways to calculate the Jacobian in a numerical framework such as the FEM. Conceptually the most straightforward way is to explicitly perturb regions in the numerical model and take the limit when the pertubation approaches zero. This scheme, however, is not very efficient. Another possilibity is to take the discretized weak form of the DE with a real source $q_{o}$ as a starting point, and differentiate this with respect to a parameter value in a region (in the simplest form in one element). This results into a DE for the sensitivity values. Solving this form for each region (element), the Jacobian can be constructed. Considerable advantage in the speed of the calculation may be obtained by utilizing the reciprocity principle, stated as: The measurement of the flux at $\mathbf{r}_{m}$ that is due to an isotropic source at $\mathbf{r}_{q}$ is equal to the measurement of the photon density at $\mathbf{r}_{q}$ produced by a source at $\mathbf{r}_{m}$, provided that the source is created by an adjoint measurement operator. This leads to the so-called adjoint method (Arridge and Schweiger, 1995).

\subsubsection{Methods for Solving the Inverse Problem}

Various approaches for image reconstruction in optical tomography have been presented. An important tool in image reconstruction are perturbation approximations, which approximate the change in the field due to a change in the material parameters. Many image reconstruction algorithms are based on the first order Born or Rytov approximations. Let us consider perturbations in the material properties: $\mu_{a}=\mu_{a, \mathrm{o}}+\delta \mu_{\mathrm{a}}$ and $\kappa=\kappa_{\mathrm{o}}+\delta \kappa$. The Born approximation assumes that the perturbed field is obtained as:

$$
\Phi=\Phi_{0}+\Delta \Phi
$$

where $\Phi_{0}$ is the field for unperturbed parameters $\mu_{a, 0}, \kappa_{0}$ and the perturbation $\Delta \Phi$ in the field is obtained as:

$$
\Delta \Phi=\int_{\Omega} \delta \kappa\left(\mathbf{r}^{\prime}\right) \nabla_{\mathbf{r}^{\prime}} G_{0}\left(\mathbf{r}, \mathbf{r}^{\prime}, \omega\right) \cdot \nabla_{\mathbf{r}^{\prime}} \Phi\left(\mathbf{r}^{\prime}, \omega\right)+\delta \mu_{a}\left(\mathbf{r}^{\prime}\right) G_{0}\left(\mathbf{r}, \mathbf{r}^{\prime}, \omega\right) \Phi\left(\mathbf{r}^{\prime}, \omega\right) d \mathbf{r}^{\prime} .
$$

The Rytov approximation assumes

$$
\operatorname{In} \Phi=\operatorname{In} \Phi_{0}+\delta u
$$

for the perturbed field $\Phi$, where $\delta u$ is obtained as

$$
\delta u=\frac{-1}{\Phi_{0}(\mathbf{r}, \omega)} \int_{\Omega} \delta \kappa\left(\mathbf{r}^{\prime}\right) \Delta_{\mathbf{r}^{\prime}} G_{0}\left(\mathbf{r}, \mathbf{r}^{\prime}, \omega\right) \cdot \nabla_{\mathbf{r}^{\prime}} \Phi\left(\mathbf{r}^{\prime}, \omega\right)+\delta \mu_{a}\left(\mathbf{r}^{\prime}\right) G_{0}\left(\mathbf{r}, \mathbf{r}^{\prime}, \omega\right) \Phi\left(\mathbf{r}^{\prime}, \omega\right) d \mathbf{r}^{\prime} .
$$

In the following, we shall outline some popular methods for image reconstruction. 
3.4.4a. Analytical inversion. In (Markel and Schotland, 2001), analytical inversion formulas based on the FDDE were derived. In this analysis, small arbitrary-shaped perturbations $\delta \mu_{a}(\mathbf{r})$ and $\delta \kappa(\mathbf{r})$ on $\mu_{a}$ and $\kappa$, respectively, in a homogeneous background are considered. The difference in the boundary data due to these perturbations is written using the perturbation theory and Green's functions. Using the explicit forms of the analytical Green's functions in different geometries (planar, cylindrical, spherical), the boundary data function can be written in a form of a Fourier-Laplace transform of the perturbations $\delta \mu_{a}(\mathbf{r})$ or $\delta \kappa(\mathbf{r})$. Formal inversion of the transformation then leads to an inversion formula for the perturbation. These formulas can be used to implement a practical image reconstruction algorithm.

3.4.4b. Backprojection and Backpropagation. One approach for image reconstruction is to directly apply conventional computed tomography (CT) backprojection algorithms. In (Walker et al., 1997), a filtered backprojection algorithm using a straight line weight function (as in X-ray CT) was applied for optical measurements. The backprojection approach can be improved by using weight functions that take into account the diffuse propagation of light in turbid media.

The backpropagation approach can be developed based on diffuse light propagation and a linearizing approximation. In (Matson and Liu, 1999), a backpropagation algorithm was developed by writing an expression for the scattered wave produced by pertubations in $\mu_{a}$ and $\mu_{s}^{\prime}$. Under the Born approximation, the scattered wave was written as an integral over the perturbed domain involving the homogeneous solution to the wave equation and the appropriate Green functions.

The backpropagated wave inside the medium is then reconstructed by back-propagating the detected wave througout the turbid medium. Studying the explicit formulas for reconstructed waves, the authors note that the reconstructed wave is what would be obtained if the scattered wave would actually be measured in the reconstructed plane and that the planes of reconstructed wave can be used to localize objects. More complicated inhomogeneous structures could be reconstructed using a multiple-view backpropagation algorithm, which consisted of first reconstructing the backpropagated waves from all views used for measurement, and then coherently summing the backpropagated waves.

The advantage of backprojection and backpropagation algorithms is their speed, especially when the $3 \mathrm{D}$ case is concerned. They can provide structural information and have been suggested to be used as nearly real-time imaging algorithms. The results of these algorithms could also be used as a starting point for a more robust or quantitative reconstruction algorithm.

3.4.4c. Linear Methods. Linear single-step reconstruction methods can be developed based on integral or differential formulation of the first order perturbation approximation.

A linearized method using integral formulation (see e.g. O'Leary et al., 1995) is obtained assuming that the perturbed field due to perturbations $\delta \mu_{a}$ and $\delta \kappa$ is obtained as a first order Born approximation $\Delta \Phi_{1}$ by replacing $\Phi$ by $\Phi_{0}$ on the right-hand side of Eq. (56). The resulting integral equation can then be discretized by rewriting the integral as a sum over voxels, leading to a set of coupled linear equations. This set can also be written in a matrix form $y=A x$, where $y$ is a vector containing measurements and $x$ the optical parameter values in the image voxels, and then solved by matrix inversion or algebraic reconstruction techniques. 
The differential formulation can be derived by expanding the forward model (Eq. (8)) as a Taylor series at point $p_{0}$, that is close to the ideal solution,

$$
y=F\left(p_{0}\right)+F^{\prime}\left(p_{0}\right)\left(p-p_{0}\right)+\left(p-p_{0}\right)^{T} F^{\prime \prime}\left(p_{0}\right)\left(p-p_{0}\right)+\ldots,
$$

and neglecting the terms after first order. In the discrete case the term $F^{\prime}$ is represented by the Jacobian matrix $\mathcal{J}_{p}^{y}$ defined in Eq. (51). Writing $p-p_{0}=\delta p$ and $y-F\left(p_{0}\right)=$ $y-y_{0}=\delta y$ we arrive at:

$$
\delta y=\mathcal{J}_{p}^{y} \delta p .
$$

The discretization is usually done using the finite difference or finite element methods. The matrix equation (60) is often ill-posed and large. Various methods have been used to solve this equation, including the truncated singular value decomposition (SVD) or the algebraic reconstruction technique (ART).

The linear perturbation method is valid only for small perturbations. Commonly, especially if quantitative reconstruction is desired, nonlinear methods are used.

3.4.4d. Nonlinear Optimization Methods. In (Ostermeyer and Jacques, 1997), an iterative reconstruction approach based on the integral formulation was presented. The method used the first-order Born integral formulation and proceeded by iterating successive higher order approximations $\Phi_{n}$ for $\Phi$ using the previously obtained estimate $\Phi_{n-1}$ in the integrand.

Most nonlinear optimization methods aim to minimize an objective function involving the measured data and the forward model between the data and the estimated parameters of a form

$$
\mathcal{G}(p)=\|L(y-F(p))\|^{2},
$$

where $L$ is a weighting matrix. Often a diagonal data scaling vector is used, e.g., $L(i, i)=\mathrm{I} / \sigma_{i}$, where $\sigma_{i}$ is the standard deviation of the measurement.

Two popular types of optimizaton schemes are now considered (the discussion here follows Kolehmainen (2001) and Arridge and Schweiger (1998), see the references for details). First, Newton-type methods seek to minimize $\mathcal{G}(p)$ iteratively using the Taylor expansion of $\mathcal{G}(p)$ around the current estimate $p^{(\kappa)}$. The new estimate is obtained as

$$
p^{(\kappa+1)}=p^{(\kappa)}-\left[\frac{\partial^{2} \mathcal{G}}{\partial p^{2}}\left(p^{(\kappa)}\right)\right]^{-1} \frac{\partial \mathcal{G}}{\partial p}\left(p^{(\kappa)}\right) .
$$

Generally, this leads to a Newton-Raphson iteration involving a second-order derivative $\partial^{2} F / \partial p^{2}$. In practical situations, the computation of the second order term may become too slow, so approximations to the Newton-Raphson method are needed. Two popular approximations are the Gauss-Newton method and Levenberg-Marquardt -type iteration. Denoting $\partial F / \partial p\left(p^{\kappa}\right)=\mathcal{J}_{(\kappa)}$ and $L^{T} L=W$, the Gauss-Newton iteration formula becomes

$$
p^{(\kappa+1)}=p^{(\kappa)}+s_{(\kappa)}\left(\mathcal{J}_{(\kappa)}^{T} W \mathcal{J}_{(\kappa)}\right)^{-1} W\left(y-F\left(p^{(\kappa)}\right)\right) .
$$

The Levenberg-Marquart iteration involves a control term $\lambda I$ :

$$
p^{(\kappa+1)}=p^{(\kappa)}+s_{(\kappa)}\left(\mathcal{J}_{(\kappa)}^{T} W \mathcal{J}_{(\kappa)}+\lambda I\right)^{-1} W\left(y-F\left(p^{(\kappa)}\right)\right) .
$$

where $\lambda \geq 0$ is a control parameter. 
A second type of optimization schemes considered here are the gradient methods, which are preferable when the dimension of the optimization problem becomes very large. A prototype gradient method is the conjugate-gradient method, where a set of search directions is generated to minimize the objective function. The minimum is searched by an iteration where the objective function is minimized along the current search direction using one-dimensional line minimization and after that a new seach direction is generated. The procedure is repeated until a termination criterion is satisfied. In conjugategradient method, a new search direction $d^{(n+1)}$ is obtained as a weighted sum from the previous search direction $d^{(n)}$ and the gradient $z$ of the objective function at the current estimate:

$$
d^{(n+1)}=-z\left(p^{(n+1)}\right)+\beta^{(n+1)} d^{(n)},
$$

where $z\left(p^{(n+1)}\right)=\partial \mathcal{G} / \partial p=-2 W \mathcal{J}^{T}\left(y-F\left(p^{(n+1)}\right)\right)$ and $\beta$ determines the weighting.

Due to the ill-posedness of the inverse problem, often regularization is introduced. A commonly used regularization method is Tikhonov type regularization. In generalized Tikhonov regularization, the objective function to be minimized becomes

$$
\mathcal{G}(p)=\| L\left(y-F(p) \|^{2}+\alpha A(p),\right.
$$

where $A(p)$ is regularizing function and $\alpha \geq 0$. For Newton-type optimization, a commonly used method is Tikhonov regularized Gauss-Newton iteration:

$$
p^{(k+1)}=p^{(k)}+s_{(k)}\left(\mathcal{J}_{(k)}^{T} W \mathcal{J}_{(k)}+\frac{1}{2} \alpha H_{A}^{(k)}\right)^{-1}\left(\mathcal{J}_{(k)}^{T} W\left(y-F\left(p^{(k)}\right)\right)-\frac{1}{2} \alpha g_{A}^{(k)}\right),
$$

where $H_{A}^{(k)}=\partial^{2} A / \partial p^{2}\left(p^{(k)}\right)$ and $g_{A}^{(k)}=\partial A / \partial p\left(p^{(k)}\right)$. For the gradient methods, the gradient methods, the gradient $z$ is modified to $z=-2 W \mathcal{J}^{T}(y-F(p))+\alpha \partial A / \partial p$.

3.4.4e. Statistical Methods. Recently, statistical inversion methods have received some attention in the field of optical imaging (Kolehmainen, 2001; Ye et al., 1999). In the statistical approach, the measurables and the parameters are treated as quantities that are either directly observable (measurables) or cannot be directly observed (parameters). These quantities are modelled as random variables with their respective probability densities, which can be joint as the quantities depend on each other through a more or less well known model. One interpretation of this method is that the uncertainty in parameter values is modeled through probability distributions. The solution to the inverse problem is the posterior probability density of the parameters in condition of the obtained measurements. This posterior density is derived using all available information on the measurements, the underlying model and prior information. Often point estimates, such as maximum a posteriori estimate, are drawn from the posterior density to obtain more intelligible solution, which in many cases involves tedious computing (Kaipio et al., 2000).

\subsubsection{Absolute and Difference Imaging}

Two different imaging strategies often identified are absolute imaging and difference imaging. Absolute imaging comprises a full modeling of the internal distributions of scattering and absorption of the object to be imaged and attempts to reconstruct the true distributions of absorption and/or scattering. In difference imaging, two states of the object 
are compared, i.e., two data sets are obtained, one before and one after a change in the object, and image reconstruction aims to produce an image that reveals these changes. In practice, difference imaging is an easier approach than absolute imaging, and hence the more commonly applied one. Also, if time-varying data is measured, dynamic features of the states can be accessed (Barbour et al., 2001).

\subsubsection{D Imaging}

Nowadays 3D image reconstruction schemes are becoming increasingly common. As true measurements are always performed in $3 \mathrm{D}$, realistic modeling and image reconstruction requires a full 3D approach. The requirements for the memory and the computational power are substantially higher in 3D, as the dimension of the discretized problem grows rapidly. Considering, e.g., FD or FE based modeling approaches, efficient matrix manipulation and inversion schemes become of great importance. For the commonly used FE modeling, another 3D challenge is mesh generation. Mesh generation, as well as checking the validity of the generated meshes, becomes essentially harder in 3D. Yet another complication in 3D is the visualization of the parameter distributions and solutions.

\section{INSTRUMENTATION AND EXPERIMENTAL METHODS}

In optical imaging, the instruments can be classified into three categories by the type of data they provide: the continuous wave $(\mathrm{CW}, \mathrm{DC})$ method in which the intensity is measured, the frequency domain method (FD) in which the light is modulated and the amplitude and phase shift are measured, and the time domain method (TD) in which the full time-of-flight distribution of the photons is measured.

\subsection{Instrumentation for the Measurement of DC Intensity}

The measurement of intensity (corresponding to Eq. 36 at $\omega=0$ ) can be performed with relatively simple continuous-wave light sources and detectors. To include several wavelengths and light source positions, either modulated or pulsed light is used.

\subsubsection{Modulated Systems}

Intensity modulation of the light source and subsequent amplitude measurement has the advantage of reduced low-frequency-noise. This includes $1 / \mathrm{f}$ noise in the electronics and stray light from external light sources. (Fig. 4.)

Multiple wavelengths can be implemented by encoding them with different modulation frequencies, or by time-multiplexing.

If a fully parallel measurement is desired, each source can be assigned a different modulation frequency. However, the system complexity increases quickly with the number of optodes, and the dynamic range is compromised. This technique is most useful for dynamic two-dimensional imaging of the cortex. 


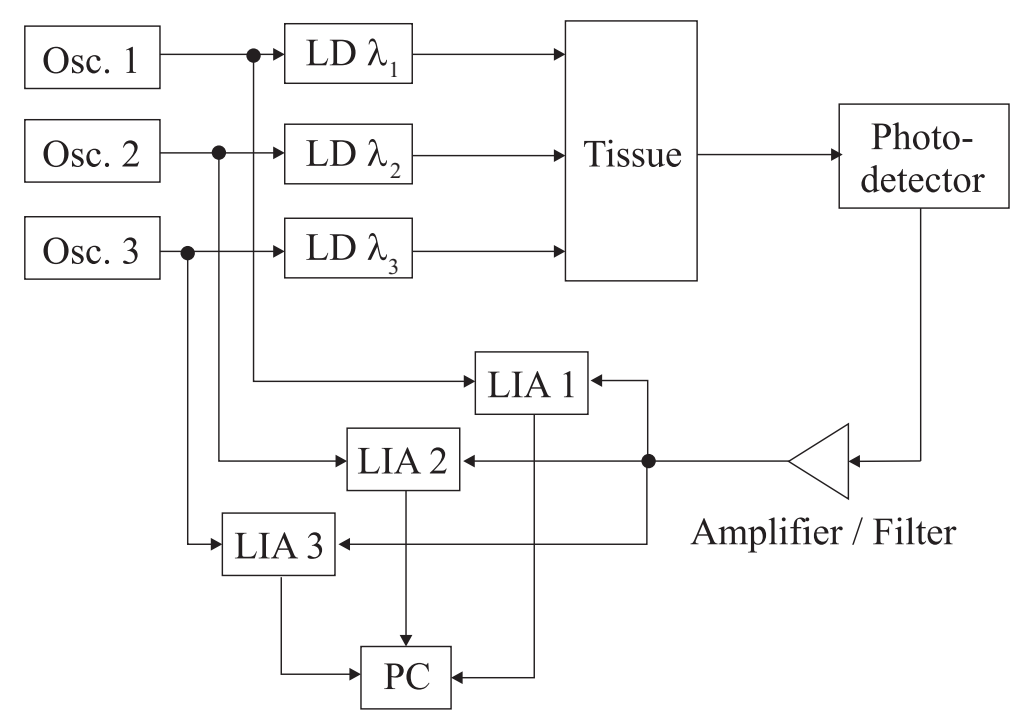

Figure 4. An intensity-modulated continuous-wave instrument with three wavelengths. Osc. $=$ oscillator, $L D=$ laser diode, LIA = lock-in amplifier, $\mathrm{PC}=$ personal computer.

\subsubsection{Time-Multiplexed Systems}

To solve the problem of dynamic range in optical tomography, time-multiplexing of the light sources is necessary.

A laser diode can be switched on and off using the injection current, and this can be combined with modulation if necessary. Another possibility is to use mechanical shutters for each laser diode. This has the advantage of a high switching speed $(\sim 1 \mathrm{~ms})$. A disadvantage is the large number of laser diodes required in a tomographic system. If only one laser diode is used for each wavelength, the light can be directed to the active source fiber using a fiberoptic or a mirror-based switch (DiCon FiberOptics, 1998; Schmitz et al., 2002). A time-multiplexed system is illustrated in Fig. 5.

\subsubsection{Detectors in Continuous-Wave Systems}

Most NIR-sensitive detectors can be used in continous-wave systems. Speed is usually not a problem, although there are a few detector types which are too slow for modulated light $(\sim \mathrm{kHz})$. In applications which require relatively small interoptode distances, silicon photodiodes (SiPD) are typically used. The high quantum efficiency $(\sim 70 \%)$ results in a high signal-to-noise ratio and these detectors are not easily damaged by excessive incident light. If high speed is not required, detectors with a large sensitive area $\left(>10 \mathrm{~mm}^{2}\right)$ may be used. A disadvantage of SiPDs is that normally they have no integrated current amplification, and thus noise of the subsequent electronics may overwhelm the dark current, increasing the detection limit of the system. Newer detectors with integrated amplifiers are available, but their speed at a given photosensitive area is not as good. To increase dynamic range, gain-programmable amplifiers can be included (Schmitz et al., 2002).

At very low $(<1 \mathrm{pW})$ light levels, photomultiplier tubes (PMTs) are usually the best choice of detector. They use a secondary emission cascade to increase the current from a 


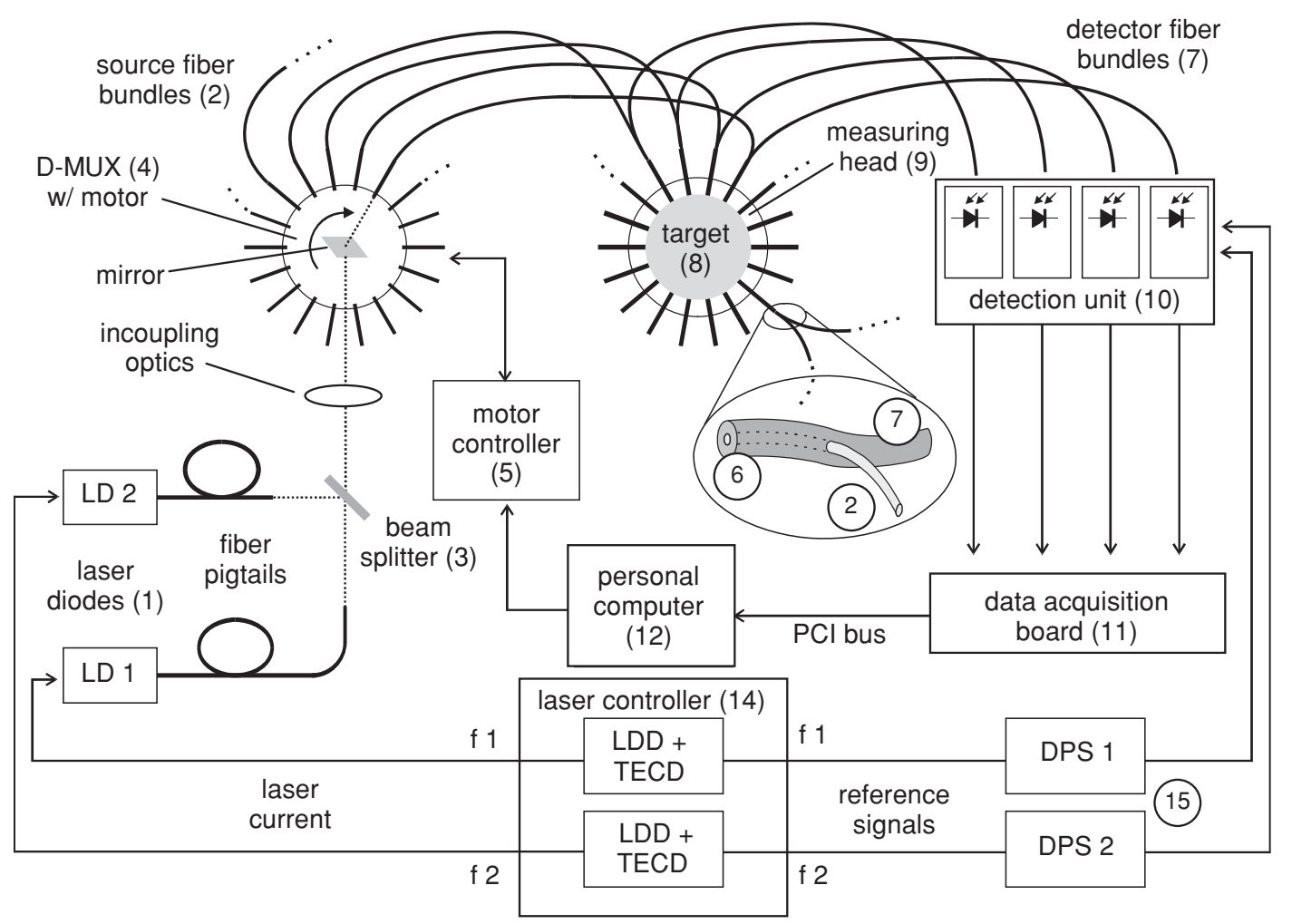

Figure 5. A continuous wave instrument with time-multiplexed source fibers. $\mathrm{LDD}=$ laser diode driver, TECD = thermoelectric cooling driver, f1, f2: laser diode modulation frequencies, LD1, LD2: laser diodes, D-MUX: optical demultiplexer, DPS1, DPS2: digital phase shifters. Reproduced with permission from (Schmitz et al., 2002).

single detected photon. PMTs have a relatively low quantum efficiency for near infrared light $(\leq 10 \%)$, but they have a very low dark current and the noise figure of the dynode chain is very low $(\sim 1.2)$. They are somewhat vulnerable to high intensities and anode currents. The photosensitive area is quite large $\left(\geq 50 \mathrm{~mm}^{2}\right)$ and they can be made with a fast response time (electron transit time spread (TTS) $\sim 200 \mathrm{ps}$ ). Due to the relatively large size and the high voltage required, PMTs are not ideal for direct-contact measurements with tissue.

The instrumentation is compact and simple enough for the implementation of multichannel systems which can be placed on the surface of the tissue without optical fibers (Vaithianathan et al., 2002). The light sources and large-area photodiodes can be (almost) in direct contact with the tissue, yielding a very high signal-to-noise ratio.

\subsubsection{Light Sources}

Laser diodes and light-emitting diodes (LEDs) are used in CW systems. Laser diodes have a narrower spectral bandwidth and they can be driven with lower electrical current than LEDs, thus they are usually preferred.

\subsubsection{Calibration and the Use of Data}

The separation of scattering and absorption in general requires the measurement of intensity along with at least one other feature of the TPSF, such as the mean time. Thus, while 
the changes in the measured intensities reflect both changes in scattering and absorption, it is not possible to identify these changes as one or the other based on intensity alone (Arridge and Lionheart, 1998). Usually, it is assumed that scattering is not time-varying and changes in the absorption coefficient can then be spatially resolved based on the measured intensities. Since the background optical properties cannot be uniquely determined, the initial assumption is that the medium is homogeneous.

Intensity provides a high contrast-to-noise ratio to changes in the absorption of tissues close to the surface. Unfortunately, it is also very sensitive to the contact between the optodes and tissue. To reduce the variability of the contact, it may be preferable to attach the optodes at a distance of a few millimeters from the skin. If dark hair is present, a contact measurement may be a good choice, as it reduces the motion of hair under the optodes. A pressure sensor can in some cases be used to measure changes in the contact.

Intensity data is usually not explicitly calibrated, instead, a reference measurement is made. The reference can be a phantom with known optical properties and geometry. In the ideal case, the shape and dimensions of the reference are exactly the same as that of the tissue. The differences between the logarithms of intensities in the two states (reference and tissue) are used to reconstruct a map of the differences in the optical properties between the two states.

If desired, a calibration of the data can be performed with a homogeneous cylindrical phantom. By measuring the data corresponding to each source-detector pair, the coupling coefficients and the differences between the channels can be resolved based on symmetry. (Schmitz et al., 2000) It is also possible to solve for the calibration parameters together with the optical properties in the reconstruction (Boas et al., 2001a).

\subsection{Instrumentation for the Measurement of the TPSF}

The most flexible of the three instrument types is the time-domain instrument, which measures the flight time of each detected photon emitted by a pulsed light source.

\subsubsection{Measurement Principle}

In a time-resolved instrument, the light is emitted to the tissue in a rapid train $(\sim 100 \mathrm{MHz})$ of very short pulses $(1-100 \mathrm{ps})$. The photons that are transmitted through the tissue are detected at several locations on the surface of the tissue. The signal for each detected photon is amplified in the PMT and preamplifier. The resulting electrical pulse is timed using a constant fraction discriminator (CFD) and a unit which compares the timing of the CFD-generated TTL pulse and the earlier electrical pulse produced by the pulsed laser. The time-comparison is usually realized with a time-to-amplitude converter (TAC) or a picosecond time analyzer (PTA). The pulses are then added to a histogram in digital electronics. The histogram of the photon flight times is then the uncalibrated TPSF. (Fig. 6.)

An advantage of the photon-counting technique is the possibility of selecting only those analog pulses, the heights of which are within a preselected range. The pulse heights due to cosmic rays is higher than average. Lower-energy pulses are likely to be the result of thermal excitation, while the remaining pulses are likely to be caused by true photoelectron emissions in the PMT. 


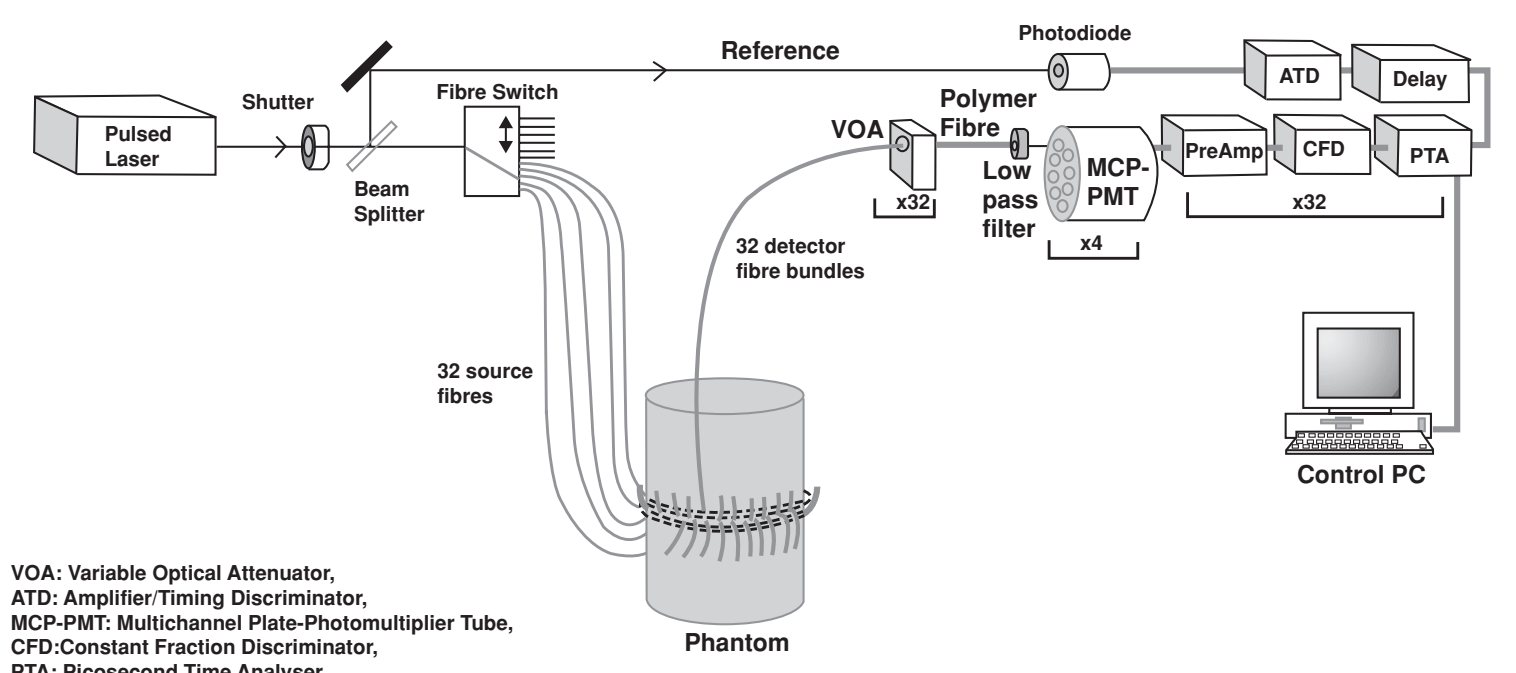

Figure 6. A time-resolved instrument (Schmidt et al., 2000). Reproduced with permission from (Hillman, 2002).

\subsubsection{Detectors in Time-Resolved Systems}

Since the electron transit time spread in PMTs is of the same order of magnitude as the TPSF, a fast PMT should be used to minimize distortion of the TPSF shape. Metal-channel PMTs such as the Hamamatsu R7400 series have a TTS of $\sim 230$ ps, and microchannel plate (MCP) PMTs have a TTS of $\sim 45$ ps. MCP-PMTs can be implemented with multiple anodes but interchannel crosstalk and high cost limit the use of this detector type in imaging systems.

Photon-counting systems have a limited dynamic range; the photon count rate is limited by the electronics to a value which is usually under $10^{6}$ counts per second (cps). While this is high enough for large interoptode distances in a transmission geometry, it limits the signal-to-noise ratio available in the reflection geometry. For example, a typical intensity detected in activation imaging with a $3-\mathrm{cm}$ interoptode distance is $50 \mathrm{pW}$ (depending on the source power, hair, the diameter, and the numerical aperture of the optical fiber bundles), which corresponds to approximately $20 \cdot 10^{6} \mathrm{cps}$.

In order to reduce the intensity that can reach the detector, a variable optical attenuator (VOA) can be used to attenuate light in a controlled way (Eda et al., 1999; Schmidt et al., 2000). It consists of a disk with a set of holes of different sizes, or neutral density filters with different optical densities. The attenuation is controlled with a stepping motor. A VOA increases the time-multiplexing time and may affect the reproducability of the measurements. Also, the calibration of the instrument becomes more difficult. In a pure transmission geometry, which is sometimes used in breast imaging, the required optical dynamic range may be small enough so that attenuation is not necessary even in a time-resolved system.

\subsubsection{Light Sources}

Time-resolved imaging systems use a pulsed light source, generally capable of emitting pulses in the range from 1 ps to 100 ps. Typically, the average power is about $10 \mathrm{~mW}$, 
although some systems operate at powers below $0.1 \mathrm{~mW}$ to comply with FDA's eye-safe classification (Ntziachristos et al., 1998).

In laboratory conditions, mode-locked picosecond Ti:Sapphire lasers are often used, but they are impractical for clinical use. Pulsed laser diodes can be used to generate $\sim 50$-ps pulses, which may be adequate with appropriate calibration. Mode-locked and frequencydoubled fiber lasers (manufactured by IMRA America) are also available. They are potentially more stable and compact than Ti:Sapphire systems.

\subsubsection{Calibration}

The TPSF is distorted to some extent by the instrument. The optical fibers have temporal dispersion, the detector has a finite TTS and the finite laser pulse width also contributes to the instrument response function (IRF). If the full TPSF is used for reconstruction, it is necessary to measure the IRF and correct the measured TPSF by deconvolution. If only data types such as mean time, integrated intensity, variance and the Laplacian are used, a simpler calibration procedure can be used (Hillman et al., 2000). For example, the mean time is shifted by a constant (equal to the mean time of the IRF), and this is true for the variance of the TPSF as well. The normalized Laplace transform can be calibrated by dividing it by the transform of the IRF.

In practice, calibration is only needed for absolute (static) imaging of the optical properties. Calibration is usually performed in steps. Absolute calibration of temporal data types can be performed using a tool which holds the source fiber and detector fiber bundle at a fixed distance from each other. A diffusing paper is used between them to excite all the modes in the multimode detection fiber bundle. The tool should not produce temporal broadening of the pulse. Interchannel variations in the IRF can be measured using a separate relative calibration tool or simply by measuring each fiber in the absolute calibration tool.

If the source and detector fibers are combined into a single optode, it is possible to perform a part of the calibration in situ. Each source is switched on sequentially to illuminate the surface of the tissue under the corresponding detector fiber bundle. The TPSF measured is an approximation of the IRF of the instrument. The lengths of the source fibers must be measured separately. The diffuse reflection by the tissue and the characteristics of the surface should be accounted for. Since the procedure can be performed on-line, interchannel drift is reduced. (Hebden et al., 2003).

If a reference phantom with identical dimensions to the tissue to be imaged exists, and if its optical properties are known, it is possible to avoid explicit calibration by reconstructing difference images based on the differences in the data measured on the tissue vs. the data measured on the phantom. If a nonlinear reconstruction algorithm is used, the resulting images should be equivalent to those obtained with absolute calibration.

Another way to use the time-resolved data is to divide the TPSF into temporal windows and look at the intensity of light within each window. This allows the selection of photons that have travelled a short path or those that have travelled longer ones. The earlier photons travel more straightforward paths while the later ones have spread out more in the tissue. This can be used to estimate scattering and absorption. In a reflection geometry, the later photons provide higher sensitivity to changes in the deeper layers of tissue. (Steinbrink et al., 2001). 


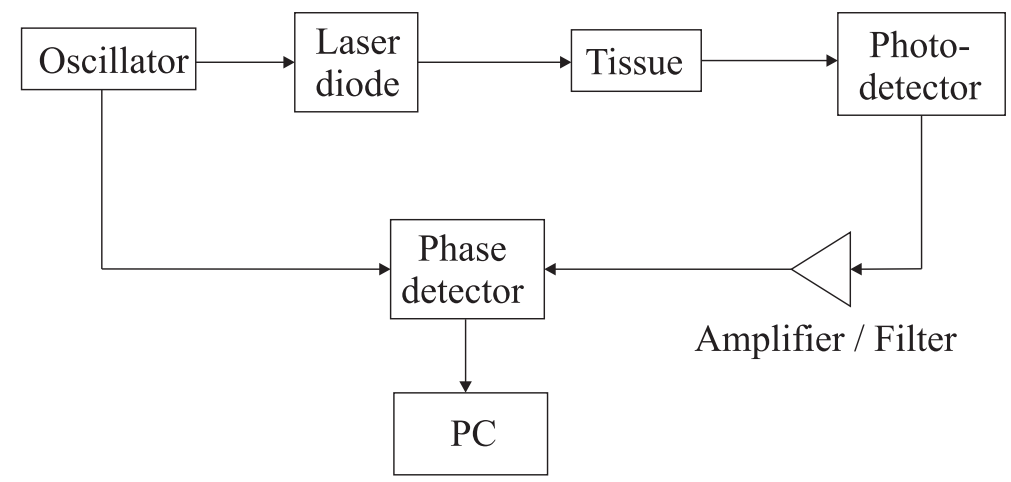

Figure 7. A homodyne frequency-domain instrument.

\subsection{Instrumentation for the Measurement of Phase and Amplitude}

In the time domain, the information of the TPSF is collected with a finite time resolution. The corresponding information may be obtained by measuring the phase and amplitude of modulated light at a set of modulation frequencies. While a few multifrequency implementations exist (Pham et al., 2000), the dynamic range and noise of a frequency-domain system can be improved by optimizing the electronics for a single frequency. The amplitude and phase at a single frequency (Eqs. 36 and 39) may be sufficient for the reconstruction of scattering and absorption images.

\subsubsection{Homodyne Systems}

In a homodyne system, the light source is modulated at a radio frequency, and the phase and amplitude of the detected and amplified signal are measured using a phase and amplitude detector, such as a lock-in amplifier (LIA) or an IQ circuit. A functional diagram of a homodyne system is shown in Fig. 7.

\subsubsection{Heterodyne Systems}

A heterodyne system consists of an RF part and an IF part. The modulation frequency $\mathrm{f}_{\text {mod }}$ is produced by an RF signal source. After the detected signal is amplified, it is downconverted to an intermediate frequency (IF, typically $\sim 10 \mathrm{kHz}$ ) with a mixer and a low-pass filter. The phase and amplitude can be measured using a LIA as usual. (Fig. 8.) The frequency that is used to drive the receiving mixer is $\mathrm{f}_{\mathrm{LO}}=\mathrm{f}_{\text {mod }}+\mathrm{f}_{\mathrm{IF}}$. To provide a synchronized reference IF signal, the signals from the two rf sources are mixed and low-pass filtered. It is possible to syncronize the two rf sources with an external clock using a phase-locked loop (PLL). If a digital LIA is used, the reference signal should be synchronized with the sampling frequency of the analog-to-digital converter (ADC) in the LIA to reduce phase noise. (Nissilä et al., 2002a).

Advantages of the heterodyne design include lower phase noise and larger dynamic range afforded by the use of digital LIA technology, and a wider range of modulation frequencies. 


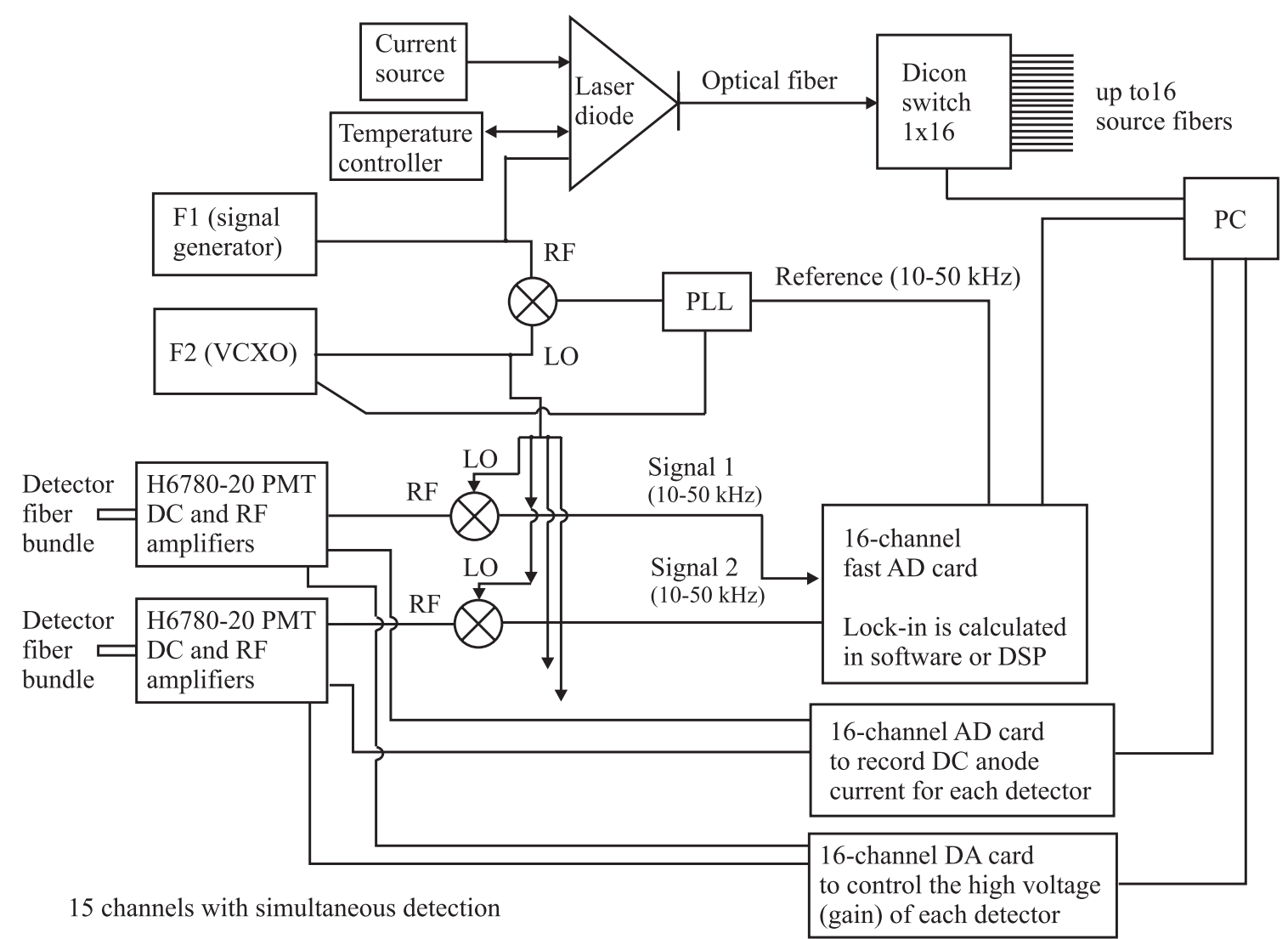

Figure 8. A heterodyne frequency-domain instrument.

\subsubsection{Phased Array}

A variation of the frequency-domain technique uses two light sources on opposite sides but at equal distances from the detector. The two light sources are modulated with a $180^{\circ}$ relative phase shift. An interference pattern in the tissue is formed by the photon density waves. The measured phase is very sensitive to changes in the optical properties of the tissue in the equidistant plane from the two sources. To scan the probed volume of tissue, it is possible to modulate the amplitudes of the light sources slowly so that their relative amplitudes change in time. This bends the plane of sensitivity. The technique has been used, e.g., in activation studies on children (Chance et al., 1998a).

\subsubsection{Detectors in Frequency-Domain Systems}

Many continuous-wave detectors can be used in frequency-domain systems. The requirements are a fast rise time and the usual high NIR sensitivity and low noise. PMTs and APDs are normally used. For optical tomography, PMTs are preferred, while APDs are excellent in applications that only require measurements at relatively short interoptode separations $(2-3 \mathrm{~cm})$. 


\subsubsection{Light Sources}

Although LEDs can also be used in a frequency-domain system, a better modulation efficiency can be obtained with laser diodes. Single-mode laser diodes are preferable, as their output is more stable against changes in temperature. Temperature stabilization should be implemented especially if the sensitivity of the photodetector depends strongly on wavelength. An impedance-matching circuit is required to obtain a good modulation efficiency.

\subsubsection{Multiplexing}

Frequency multiplexing of the light sources is not practical in the frequency domain as the number of simultaneous RF sources required is high, and the technology is already more complicated than in modulated CW systems. The different wavelengths can be frequencyencoded, however.

A practical solution is to illuminate the different source optodes sequentially. Normally, an optical switch is used for this. Since the signal-to-noise ratio of frequency-domain systems is comparable with that of DC systems, they can be used for dynamic imaging. The time constant of the LIA should be chosen so that it does not increase the effective switching time.

\subsubsection{Dynamic Range}

A difficulty in traditional phase measurement systems is that several components produce a phase shift which depends on the amplitude of the signal. This effect can be reduced by operating the components far below the top of their dynamic range. The phase-amplitude crosstalk in a photomultiplier tube depends on the relative magnitude of the anode current and the quiescent current through the voltage divider. Either by increasing the quiescent current, or by reducing the high voltage so that the anode current is much lower than the quiescent current, crosstalk can be reduced to an acceptable level.

While the crosstalk within the LIA and the crosstalk between the detection channels produce errors in measurements, these can easily be overwhelmed by the RF crosstalk between the source and the detector electronics. Both systems operate at precisely the same radio frequency, but their amplitudes can be different by as much as $170 \mathrm{~dB}$. This problem can be solved by careful radiofrequency shielding and an appropriate selection of components up to about $200 \mathrm{MHz}$. Above this frequency, it becomes increasingly difficult to preserve the dynamic range. To extend the dynamic range of the system, gain switching or VOAs can be applied (Pogue et al., 1997; McBride et al., 2001; Nissilä et al., 2002a). With gain switching, phase can be measured accurately over a dynamic range of $1: 10^{6}$.

\subsubsection{Calibration}

PMTs have an internal mechanism for gain adjustment. After the gain has been changed, there is a short transition period before the signal settles. In addition, changes 
in the intensity of the incident light also cause hysteresis and the signal is slightly delayed. If absolute measurements are required, this needs to be taken into consideration.

Calibration of frequency-domain data in the general case requires the determination of the frequency response of the instrument. If a single frequency is used, this means that the amplitude and phase of the system for an object with known optical properties is to be measured. A direct measurement of the phase delay can be made just as for the mean time in the time domain (Sect. 4.2.4). Calibration of absolute amplitude is more difficult as the contact between the optodes and the tissue as well as the surface texture of skin have a large effect on the amplitude.

If gain switching by adjusting the high voltage of the PMT is used, it is necessary to calibrate the dependency of phase and amplitude on the high voltage separately for each detector.

\subsubsection{Stability}

The temporal (or phase) stability of frequency-domain instrumentation requires special attention. Phase noise and drift of the RF system can be minimized by precise synchronization of the two RF signal sources and the LIA. The temperature fluctuations of the laser diode should be minimized without compromising the RF shielding. The photodetector is also sensitive to temperature variations, and for optimal performance it should be cooled to a stable temperature. Temperature stabilized detectors are rarely used in frequency-domain instruments due to technical difficulties in retaining a low transmitter-receiver RF coupling in such a system. The RF components should not be used close to their maximum power ratings, as phase drift increases if the temperature of the components is not stable. It is possible to reach $\sim 2 \mathrm{ps} / \mathrm{h}$ stability in a closed laboratory environment.

\subsection{Phantoms for Diffuse Optical Imaging}

Optically tissue-equivalent phantoms have been developed by several groups. Liquid phantoms are usually made by diluting Intralipid into water and adding ink to obtain the desired absorption. A problem with this approach is the mismatch in the refractive indices at glass-liquid boundaries. To create heterogeneous phantoms with a uniform refractive index, transparent resin can be used together with a dye and a scattering material, such as titanium dioxide or microspheres. The dye and scatterer are diluted in alcohol and mixed with the resin. During the hardening process, care must be taken to avoid cracks and bubbles. The anisotropy factor $g$ (Eq. 4) with $\mathrm{TiO}_{2}$ particles is about 0.5 and with latex microspheres about 0.9, which is closer to that of tissue. (Firbank et al., 1993, 1995).

\subsection{Techniques for the Attachment of Optodes on Tissue}

The coupling of optical fibers with tissue is critical for the quality of the measured data. Various techniques have been proposed, ranging from a modified motorcycle helmet to a fiberbrush. Thermoplastic materials are commonly used for head measurements. They can be reshaped for each subject and measurement area separately (Fig. 9). In other body parts, which have a thicker layer of soft tissue, it may be more difficult to obtain a stable contact 


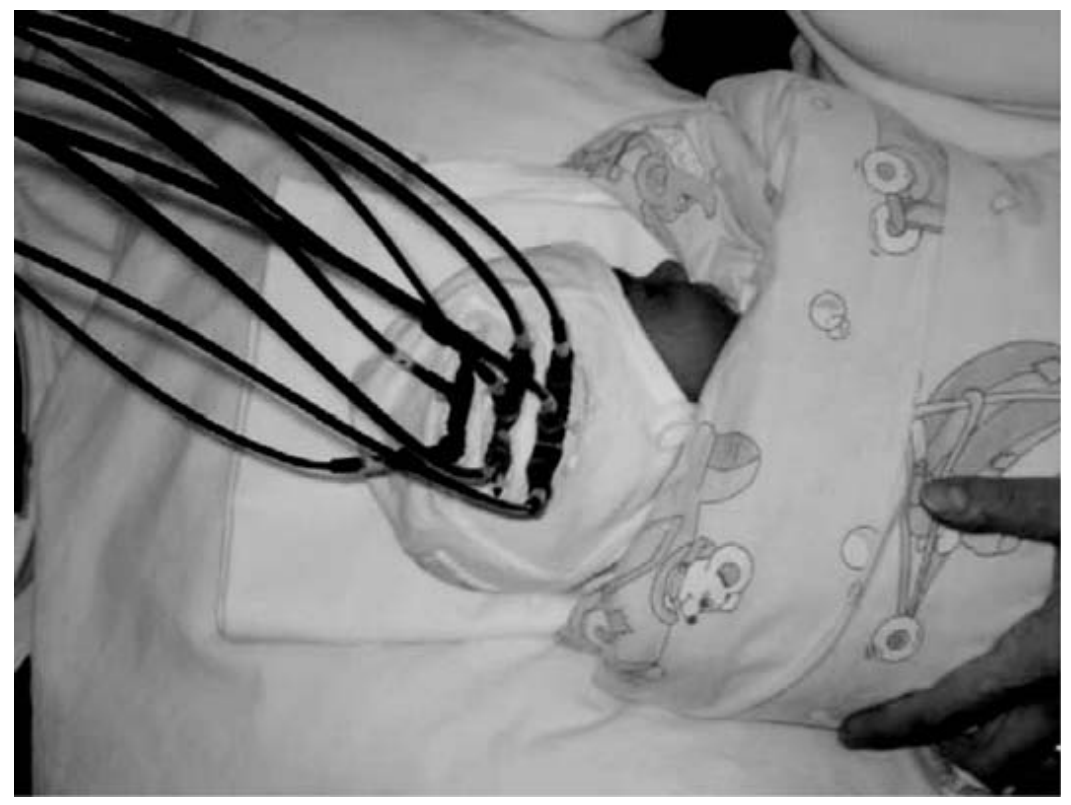

Figure 9. Optical fibers mounted on a neonate using a thermoplastic mount.

between the tissue and the optical fibers. In such cases, it may be advantageous to avoid direct contact with the tissue and let the optodes stay at a distance from the skin. In this way, the optodes do not apply pressure to the skin, which would affect circulation as well as the boundary shape. It is also possible to bend the ends of the optodes to a $90^{\circ}$ angle, which may be useful if space is constrained, such as when measuring inside a MR scanner. The angle may also help to reduce motion artifacts if the fiber bundles are attached to the body.

In the case of the head, hair is a major problem. Long and dark hair are particularly difficult. The hair may attenuate a large part of the light, which reduces the signal-to-noise ratio. The hair can also move under the optical fibers, causing large changes in the intensity of the measured signal. The temporal data types are less sensitive to this type of phenomena, but their contrast-to-noise ratio may not be sufficient to detect small changes such as those related to brain activity.

In hair-free cases, it is possible to utilize large-area SiPD detectors directly or almost directly on the surface of the tissue. This situation occurs in frontal studies with adults and often with neonates.

In optical mammography, several groups use the arrangement familiar from x-ray mammography, where the breast is compressed between two glass planes (Franceschini et al., 1997). The Philips optical mammography prototype (Colak et al., 1999) uses a scattering liquid to fill the gap between the breast and a cone containing the optodes. The optode geometry is fixed and the contact between the optodes and the liquid is constant, which should remove many artifacts. It is also possible to use precise mechanical positioners to place the optodes on the surface of the breast, as in the system by McBride et al. (2001).

\subsection{Registration}

Model-based reconstruction in optical tomography is sensitive to errors in the positions of the optodes. Markers can be placed on the skin at the optode positions, and photographed 
from different angles using a digital camera. The images are then used to reconstruct the boundary of the object. A laser-scanning system or a measurement arm which tracks the $3 \mathrm{D}$ position of the pointing head can be used to measure the shape of the object. The latter also works if hair is present. It is also possible to obtain an approximate boundary based on indirect techniques such as digitizing the surface of the thermoplastic mount that was shaped to match the subject and hold the fibers.

A FE mesh can be generated based on the recovered boundary shape. If an anatomical MR image of the subject is available, it is possible to recover the boundary (as well as boundaries between different tissue types inside) from the segmented data and use it for mesh generation. Only the optode positions (as well as the positions of markers present in the MR imaging) need to be digitized.

\section{APPLICATIONS}

\subsection{Muscle Studies}

In skeletal muscle studies, the primary aim is to investigate the oxidative metabolism and its changes. NIRS systems with a single or a few channels have been applied to muscle measurements using continuous-wave $(\mathrm{CW})$, spatially-resolved, time-domain, and frequency-domain techniques. Due to the commercial availability and lower price of instrumentation, CW studies have been most common (Ferrari et al., 1997; Boushel and Piantadosi, 2000).

Both healthy subjects and patients have been studied using single-channel continuouswave NIRS devices. Muscle experiments usually involve a study group and a control group performing the same exercise test. During or after the test, the oxygen consumption and/or recovery is monitored by recording the changes in $\left[\mathrm{HbO}_{2}\right],[\mathrm{Hb}]$, and [totHb]. The $\mathrm{CW}$ NIRS technique has also been used in general studies of oxidative metabolism in skeletal muscles and it has been applied, e.g., to sports science to investigate differences in oxygen utilization of trained and untrained subjects (Ferrari et al., 1997). The CW-NIRS studies have been validated by comparing results, e.g., with ${ }^{31} \mathrm{P}$ magnetic resonance spectroscopy (MRS) measurements, and a correlation has been found between the techniques (Sako et al, 2001). Although the single-channel CW-NIRS technique has been widely used in research, it has not been established in clinical practice because of its limitations (see Sect. 4).

The spatial mapping of oxidative metabolism of skeletal muscles gives valuable information about the redistribution of blood, local changes in blood flow, and oxygen consumption at rest and during exercise. Multi-channel CW-NIRS instruments can be used to investigate these phenomena. Such devices typically contain 12-16 channels and are capable of imaging an area of $\sim 60 \mathrm{~cm}^{2}$ (Nioka et al., 1999; Quaresima et al., 2001). The image-acquisition time of the system by Quaresima et al. (2001) is $0.1 \mathrm{~s}$, which is fast enough for imaging changes in oxidative metabolism. Even a 200-channel system with an image-acquisition time of $0.2 \mathrm{~s}$ has been developed (Niwayama et al., 2002). However, the decreased acquisition time per channel tends to reduce the signal-to-noise ratio of the data. Results from these multi-channel CW-NIRS experiments indicate, e.g., that the vasculature in general and the capillary recruitment varies locally during an exercise. Although the variations of venous blood volume and oxygen saturation are considered the principal sources 
of the optical signal, the exact contributions of different parts of vasculature are difficult to distinguish. Improvements in blood flow and venous saturation measurements using NIRS may open up new possibilities.

A typical CW-NIRS imaging system provides topographical images at a relatively low resolution. These images are often qualitative and/or relative, i.e., changes in $\left[\mathrm{HbO}_{2}\right]$, $[\mathrm{Hb}]$, and [totHb] with respect to an initial state. Utilization of tomographic reconstruction methods can provide quantitative data. A frequency-domain system has also been used to obtain topographical images using phase and amplitude data (Maris et al., 1994). With improvements in algorithms and utilization of diffusion theory, absolute values for $\mu_{a}$ and $\mu_{s}^{\prime}$ were obtained locally (de Blasi et al., 1995). From this data, the absolute concentrations of $\left[\mathrm{HbO}_{2}\right],[\mathrm{Hb}]$, and [totHb] and also the oxygen saturation were determined locally.

Using time-resolved instrumentation, a simultaneous tomographic reconstruction of $\mu_{a}$ and $\mu_{s}^{\prime}$ may be possible. A 32-channel system has been applied to study cross-sectional imaging of an adult forearm (Hillman et al., 2001). In these measurements, 32 source fibers and 32 receiving fiber bundles were fixed around the forearm of the subject. The source fibers were at a 2-mm distance from the skin, e.g., to avoid fiber-tissue coupling artifacts and the receiving bundles gently touched the skin. Absolute images revealing the optical structure of the forearm were calculated. By using two wavelengths (780/790 and $820 \mathrm{~nm}$ ), difference images showing the spatial changes in chromophore concentrations due to a finger exercise were obtained as well. The image reconstructions were carried out using a DE and FEM based algorithm in a 2D circular mesh using a 2D:3D correction for the measurement data. The mean time-of-flight data was used for the reconstructions (Fig. 10).

a) MRI
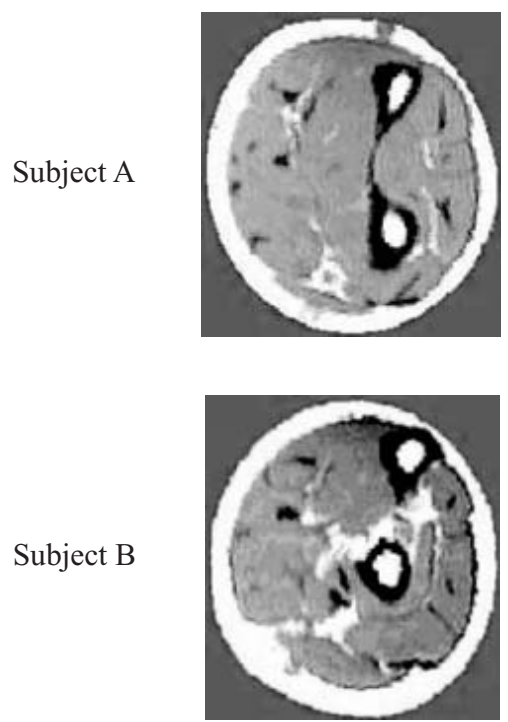

b) $\mu_{\mathrm{s}}^{\prime}$
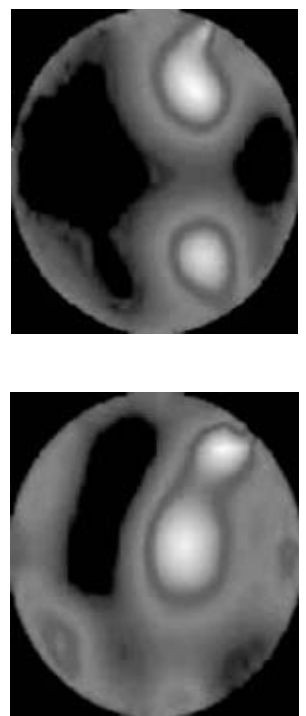

c) $\mu_{\mathrm{a}}$
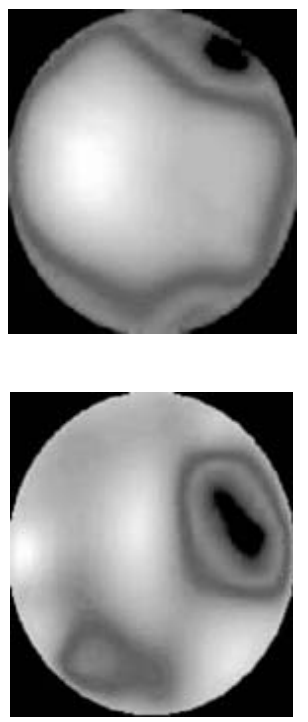

Figure 10. Absolute images of human forearms measured at $780 \mathrm{~nm}$. The image area has a diameter of $7 \mathrm{~cm}$. a) Cross-sectional MRI images of forearms of two volunteers. b) Spatial reconstruction of $\mu_{s}^{\prime}$. Bones are clearly visible because of their relatively high scattering coefficient. Also the more weakly scattering large muscles are resolved among skin and bones. c) The reconstructed absorption images correspondingly show maximum values for muscles, especially close to the largest arteries (UCL, 2001). 
Tissue heterogeneity should be taken into account in muscle studies. It is a good practice to document the thickness of the adipose layer for each measured subject and, if possible, a multi-layer model should be used in the analysis (van Beekvelt et al., 2001). Also, the location and density of blood vessels in surface tissues may influence the results, because oxygenation and hemodynamic changes can occur in the skin and subdermal tissues. It may be possible to predetermine these changes using a short source-detector separation.

Myoglobin is another oxygen-carrier molecule in muscles having absorption properties similar to those of hemoglobin but with a significantly lower concentration. The changes in myoglobin volume and oxygenation have a small effect on the measurement signal and are typically superimposed on the absorption changes of hemoglobin. Also, only the concentration changes of hemoglobin are often measured, even though cytochrome oxidase may provide additional information on the oxidative metabolism of a muscle.

Movement artifacts and geometrical changes in muscles, especially during a dynamic exercise often cause severe problems. These experimental issues might be possible to include in modeling and reconstruction algorithms. So far, mostly forearm, thigh, and calf muscles have been studied because they are easily accessible, simple to exercise, and their blood circulation can be easily manipulated. Diffuse optical imaging of other muscles, such as the abdominal, back, or neck muscles, provide another set of challenges for experiments and modeling.

\subsection{Optical Mammography}

Traditionally, clinical techniques for breast cancer imaging or mammography exploit $\mathrm{X}$-rays. The use of ionizing radiation causes a risk for the subjects and the technique has limitations regarding to its specificity and sensitivity (Moesta, 2002). MRI has also been used to diagnose breast cancer but is not in widespread use due to the cost of an examination. Optical mammography may complement the existing modalities.

Optical mammography is currently the most actively investigated application of diffuse optical imaging (OSA, 2002). Healthy breast tissue is optically relatively homogeneous, which makes it a suitable target for optical imaging. Because of its relatively low concentration of hemoglobin, it is possible to image through the whole breast. Optical mammography may offer unique information when compared with other breast imaging modalities such as X-ray, ultrasound, MRI, and isotopic imaging. Such information can be, e.g., the differences in the hemoglobin, water, and lipid concentrations and in the blood oxygen saturation between the carcinoma and healthy breast tissue. Angiogenesis and changes in the oxidative metabolism of breast tissue might be observed even before the structural changes detectable using X-rays have developed.

The Philips Medical Research Laboratory has carried out a research project using CW techniques (Colak et al., 1999). They have constructed a CW imager with 255 source fibers and 255 detector channels. In their setup, a patient lies down on an examination bed which includes a black conical cup where the optical fibers are mounted. During the imaging procedure, the breast is pendently held in the cup which is filled with a matching fluid to reduce coupling artifacts. The image reconstruction of the Philips system is based on a modified backprojection algorithm. The optical properties of the cup filled with the matching fluid are first imaged as a reference medium. Thereafter, the breast is imaged and the difference images of attenuation coefficient (i.e., $\sqrt{3 \mu_{a} \mu_{s}}$ ) are generated. In clinical 
(a)

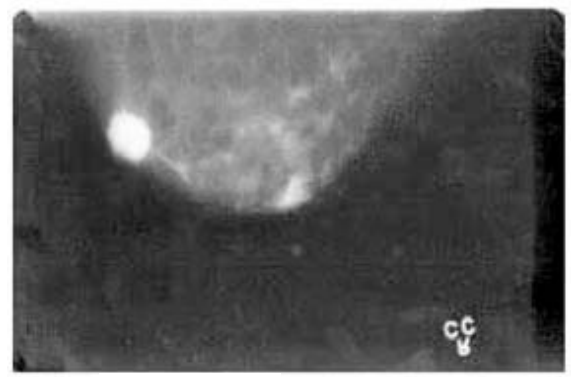
top to down

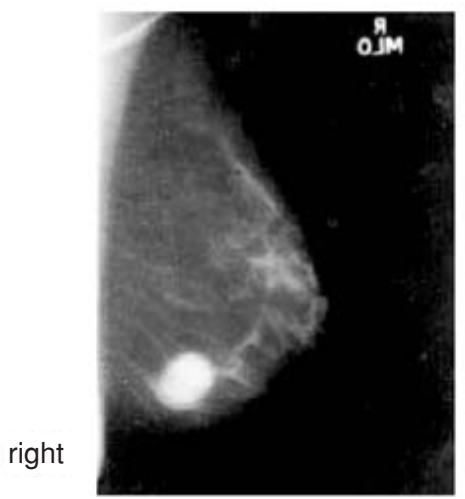

(b)

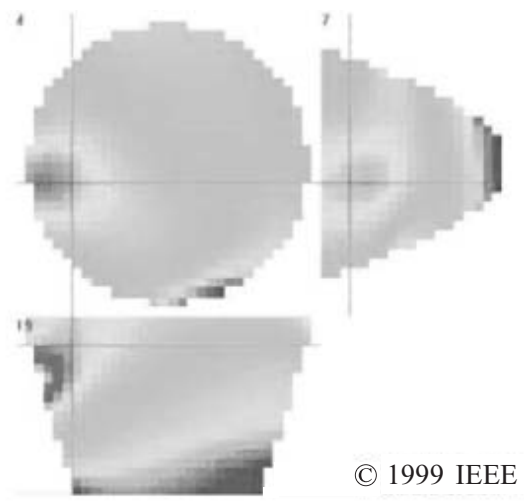

(c)

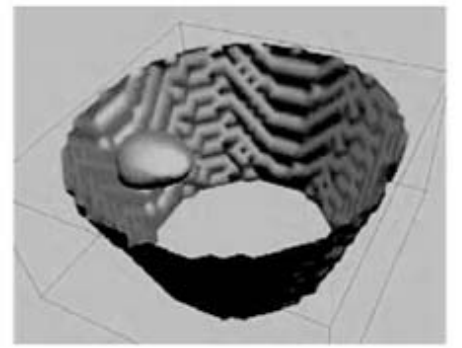

Figure 11. a) X-ray images of the right breast of a patient with Medullair carcinoma. b) Multiplanar cross-sectional optical images. The lesion is visible on the left showing a strong attenuation of light. c) A 3D isocontour image of the reconstructed cancerous tissue. The images have been reproduced with permission from (Colak et al., 1999).

tests, optical mammography was able to detect nine of the ten patients previously diagnosed to have a breast lesion using X-ray and ultrasonic imaging. In Fig. 11, a successful detection of a breast cancer is illustrated.

More comprehensive information on breast tissue can be obtained using time-and frequency-domain techniques. In the collaborative project between the Carl Zeiss company (Oberkochen, Germany) and Fantini et al., a frequency-domain instrument was used to image 69 breast cancer patients with a sensitivity of 73\%. During the measurements, the breast was compressed between two glass plates and the scan was carried out by mechanically moving the source and the detector fiberbundle on opposite sides of the breast. In the system, two imaging projections (craniocaudal and mediolateral) were possible to acquire. The system produced the so-called normalized $\mathrm{N}$-parameter maps where phase data was used for an edge correction only. In each scanning position, the parameter is related to the optical absorption of breast tissue. More recently, a similar system and algorithms have been applied to clinical tests of 131 breast cancer patients by Siemens AG, Medical Engineering (Erlangen, Germany). In these experiments, a sensitivity of $72 \%$ and specificity of $52 \%$ were obtained. (Fantini and Franceschini, 2002).

Grosenick et al. (1999) have developed a pulsed mammography system capable of measuring time-resolved transmittance images of the breast. In a typical experiment, 1500 scan positions in steps of $2.5 \mathrm{~mm}$ are collected. Normalized photoncount maps in a selected time window have been used to image the optical density of breast tissue. In addition, the $\mu_{a}$ and $\mu_{s}^{\prime}$ have been calculated from an analytical solution of the diffusion equation in 
an infinite slab assuming an optically homogeneous breast tissue. The optical properties of tumors are determined by including spherical inhomogeneities. In recent studies, random walk theory has been applied to estimate the material parameters of tumors (Grosenick et al., 2002). In clinical studies, the system has been tested using 35 suspected breast-cancer patients. The patients underwent X-ray and MRI examinations, where 26 of them were diagnosed to have breast cancer. Optical mammography was able to identify 17 cases. The nine undetected cases included a carcinoma in situ, a lymphoma, and several invasive ductal carcinomas. In some cases, the cancer was outside the imaged area but in other cases, the optical contrast between the cancerous and benign tissue was too low.

Tromberg et al. (2000) have investigated the optical properties of breast tissue, e.g., during different menopausal phases. They have developed a single-channel multi-wavelength multi-frequency instrument. In this system, the modulation frequency can be swept between $300 \mathrm{kHz}$ and $1 \mathrm{GHz}$. Both the source fiber and the APD are mounted in a hand-held probe so that the measurement head is in direct contact with the patient. The optical parameters are resolved by fitting the measured amplitude and phase values to an analytical diffusion-equation model. From the resolved absorption coefficients at each wavelength, the concentrations of the four major absorbers in breast tissue, $\mathrm{Hb}, \mathrm{HbO}_{2}$, water, and lipids are determined. The studies of Tromberg et al. have shown that the absorption and scattering coefficients of premenopausal breast tissue are typically larger than the values of postmenopausal tissue. Furthermore, they have shown that cancerous tissue may have 1.25 to 3 times higher absorption coefficient compared to healthy tissue, which should give rise to contrast in optical mammography (Tromberg et al., 2000). In a recent study of 30 healthy women and a woman with fibroadenoma, the group has shown that the multiwavelength measurement provides additional information to distinguish between normal and cancerous tissue. They demonstrated that the concentration changes of water and lipids might be better indicators of cancer than, e.g., the hemoglobin concentration or oxygen saturation (Cerussi et al., 2002).

The relatively poor spatial resolution of optical mammography has been attempted to overcome using multimodal imaging systems. The unique functional information of optical mammography has been coupled to spatially accurate X-ray and MRI images (Boas, 2002; Ntziachristos et al., 2002). In these approaches, the physiological data provided by optical mammography can be simultaneously registered with the anatomical data or, alternatively, the anatomical data can be used to guide the cancer detection in optical imaging. Also the optical contrast between a lesion and normal tissue can be enhanced using an absorbing contrast agent (Ntziachristos et al., 2000). Hybrid techniques employing fluorescent contrast agents (Hawrysz and Sevick-Muraca 2000; Ebert et al., 2001) and ultrasonic enhancement (Holboke et al., 2000) have been used to improve the sensitivity of optical mammography.

During the last few years, nonlinear inversion algorithms and FE models of the diffusion equation have been applied to in vivo data. The first published 3D FEM based reconstruction of breast tissue was based on DC data (Jiang et al., 2001) and the first FEM based reconstruction of quantitative hemoglobin concentrations in breast tissue in $2 \mathrm{D}$ was based on frequency-domain data (Pogue et al., 2001). The group at Dartmouth College has developed a frequency-domain imager with 16 time-multiplexed source channels and 16 parallel detection channels (McBride et al., 2001). The fibers are alternately fixed on a circular planar array so that the breast can be placed pendently in a central ring forming a firm contact with the tissue. 

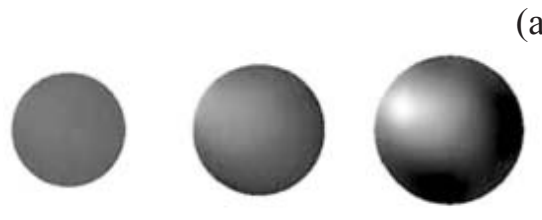

(a)
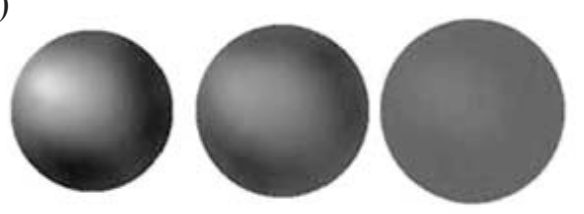

$47.71 \mu \mathrm{M}$

(b)
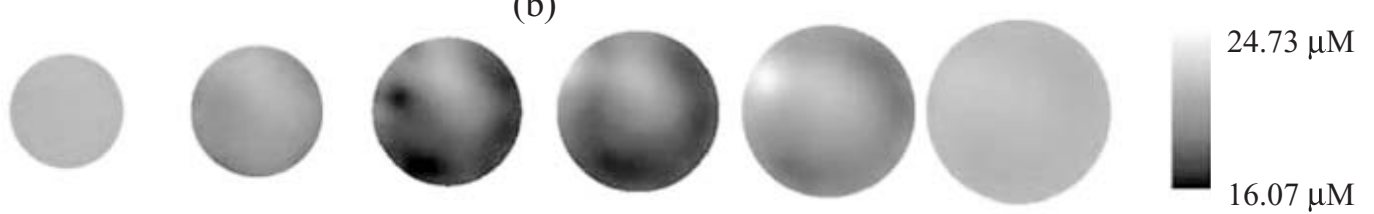

(c)
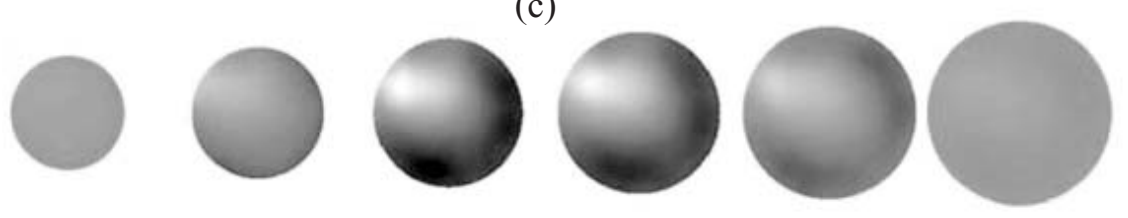

$65.28 \mu \mathrm{M}$

(d)
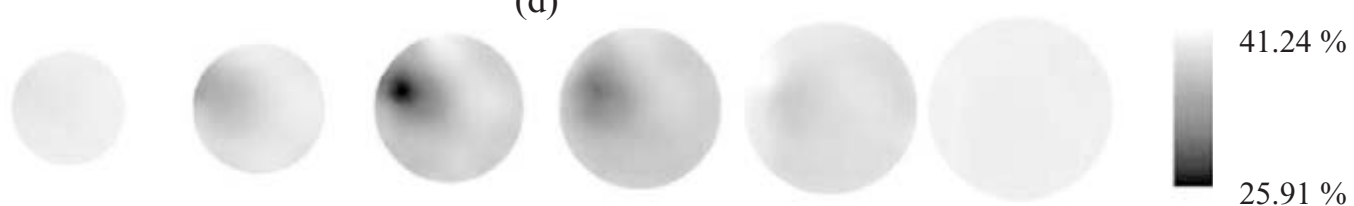

Figure 12. Maps of a) [Hb], b) $\left[\mathrm{HbO}_{2}\right]$, c) [totHb], and d) oxygen saturation. Each slice represents a different plane of the reconstruction mesh. In each case, cancerous tissue is clearly resolved in the third slice. The concentration of total hemoglobin is increased within the tumor area because of increased angiogenesis. Respectively, the oxygen saturation has decreased because of the increased oxidative metabolism due to the rapid grow of cancerous tissue. (Dehghani and Pogue, unpublished).

The Dartmouth group has recently developed a quantitative reconstruction method based on a 3D FE model (Dehghani et al., 2003). The calibration of the data at each wavelength is carried out using a reference phantom. In a recent experiment, the group imaged the breast of a subject with a malignant tumor of $11 \mathrm{~mm}$ in diameter. The phase and amplitude data were collected at four wavelengths from three planar slices (at the tumor site, at $12 \mathrm{~mm}$ above, and at $5 \mathrm{~mm}$ below the central slice). From the spatial distributions of absorption coefficient, maps of $[\mathrm{Hb}],\left[\mathrm{HbO}_{2}\right]$, [tot $\left.\mathrm{Hb}\right]$, and tissue oxygen saturation were obtained (Fig. 12).

\subsection{Optical Imaging of the Brain}

\subsubsection{Activation Studies}

5.3.1a. Introduction. Optical imaging can be used to study the hemodynamic changes induced in selective activation of the brain in a controlled activation task. Activation studies can be performed using a variety of existing brain imaging methods, such as 
multichannel electroencephalography (EEG), magnetoencephalography (MEG), functional magnetic resonance imaging (fMRI) and positron emission tomography (PET). Although both the fast activation signal (which is probably due to a change in scattering) and the hemodynamic response (which is a change in absorption) contribute to the measured signal, the hemodynamic response is much easier to detect. The spatial resolution of optical imaging is not comparable to fMRI due to the diffuse spatial sensitivity of the measurements, but optical imaging can yield a higher sample rate and the temporal resolution is only limited by the signal-to-noise ratio. Current optical topography systems can record a complete set of data for a two-dimensional image several times per second (Franceschini et al., 2000, Kohl-Bareis et al., 2002).

The instrumentation used in optical imaging is relatively compact and it can be used without expensive shielding rooms. It is applicable to bedside monitoring. Optical imaging may become an important tool for the study of infants, which are difficult subjects for other neuroimaging modalities. Another advantage is that light and low-frequency electromagnetic fields do not interfere, allowing the use of optical imaging simultaneously with other imaging modalities.

Modeling in activation studies is generally based on the assumption that the medium is optically homogeneous. Backprojection and interpolation have been used to form twodimensional activation maps (Chance et al., 1998a; Kohl-Bareis et al., 2002). The accuracy of these methods in the context of activation studies can be evaluated by comparing the images with fMRI. Phantoms can be used to study how well the algorithms work in the presence of a perturbation in a homogeneous or layered medium. The CSF layer may significantly affect the spatial sensitivity of the measurement.

5.3.1b. Imaging Paradigms. The hemodynamic response is relatively slow, reaching its maximum in approximately $5 \mathrm{~s}$. It has been found in functional MRI studies that the strongest response can be elicited by using a rapid sequence of stimuli; the repeated stimuli cause a larger total response than stimuli separated by longer intervals. The block paradigm consists of periods of rest and stimulation interleaved with each other. The whole image area is normally scanned several times within each epoch. This allows the evaluation of the time course of the response. If the image frame rate is fast enough, it may be possible to identify and partially remove the oscillations in the oxyhemoglobin signal (Sect. 2.3.) since there is a strong temporal correlation between the signals at different positions. Normally, the signal is averaged for each source-detector combination, wavelength and task condition to obtain the change in the signal caused by the task. If several wavelengths are used and the DPF is known, the modified Beer-Lambert law can be used to calculate the changes in the average concentrations of the chromophores. Unfortunately, the approach cannot be used for the quantitative measurement of localised changes in the cortex. A localised change in the hemodynamic parameters in the adult cortex is likely to be seriously underestimated due to the small fraction of the measured volume being in the cortex (Boas et al., 2001b). The sensitivity calculations used in optical tomography may be used to improve the localisation of the activation and in the estimation of the magnitude of the true change if it is assumed to be concentrated in cortical tissue. However, since transmission measurements cannot be performed with adequate spatial resolution and signal-to-noise ratio, prior information is very important in the reconstruction of activation images.

In an event-related paradigm, the hemodynamic responses to individual stimuli are measured. This allows the study of the response of the brain to changes in the stimuli. 
Using the event-related paradigm, is possible to avoid many of the problems in the block paradigm, such as low-frequency drift and oscillations. The effect of oscillations are reduced by randomizing the inter-stimulus intervals. A drawback is the loss of sensitivity as the signal is relatively weak and the time the signal is at its peak value is a small part of the total measurement time, thus prolonging the measurement time. An event-related auditory oddball study in adults was reported in (Kennan et al., 2002).

A few studies have been made, in which the event-related paradigm is applied to the fast signal presumably caused by scattering changes. The signal that can be detected transcranially is very small, and the time resolution requirement is quite high (10 ms). In principle, the spatial resolution of the scattering change should be better than that of the hemodynamic change since the spatial sensitivity of the optical measurements to scattering changes is narrower and the neural activation is more confined than the hemodynamic change. (Steinbrink et al., 2000; Gratton et al., 2001)

Of the hemodynamic parameters, the deoxyhemoglobin signal is less prone to show the oscillations in the vascular system and therefore it may provide a more robust measure of brain activity (Kohl-Bareis 2002). However, the oxyhemoglobin response is larger. Both the blood volume signal, which can be measured with a single wavelength, and the deoxyhemoglobin signal have been proposed to give the highest spatial resolution and best correspondence with brain activity of the hemodynamic signals (Culver et al., 2002; Kohl-Bareis et al., 2002).

5.3.1c. Instrumentation for Optical Activation Studies. An ideal instrument for optical topography is either a fast pulsed continuous-wave system or a frequency-multiplexed system because of their high speed and signal-to-noise ratio. Frequency-domain systems can be optimized for this application as well, with only a slight loss in signal-to-noise ratio due to the higher modulation frequency. Time-resolved systems have limited photon count rates and thus they do not give optimal signal-to-noise ratio at close source-to-detector distances. However, they can be used to differentiate between changes in different layers of the tissue by studying the different temporal windows. Photons that travel a longer time in general spend more time in cortical tissue than those that travel a short time. (Steinbrink et al., 2001)

In studies of neonates (or on the forehead of adults), it is possible to use specialized probes with large-area SiPD detectors and laser diodes or LEDs as light sources.

5.3.1d. Practical Applications. Motor, somatosensory and cognitive activity in neonates have been studied by Chen et al. (2000), Hintz et al. (2001), and Chance et al. (1998a) (Fig. 13). In the study by Kusaka et al. (2001), cerebral blood flow was measured using optical topography with a contrast agent (indocyanine green, ICG). In an asphyxiated neonate, the area of reduced blood flow was successfully localised.

In adults, motor/somatosensory, auditory, visual, and language processing have been studied. (Franceschini et al., 2000; Kennan et al., 2002; Colier et al., 2001; Obrig et al., 2002; Sato et al., 1999; Hoshi et al., 2000)

The compatibility of optical imaging with other neuroimaging methods has received wide interest. Optical measurements have been successfully combined with fMRI (Kleinschmidt et al., 1996; Toronov et al., 2001), MEG (Mackert et al., 2002), EEG (Kennan et al., 2002; Israel et al., 2002) and TMS (Oliviero et al., 1999; Iwata et al., 2000; Nissilä et al., 2002b). 
$50 \mathrm{MHz}$ Phased Array Image of Baby Brain

7-day-old premature baby $(9 / 18 / 97)$

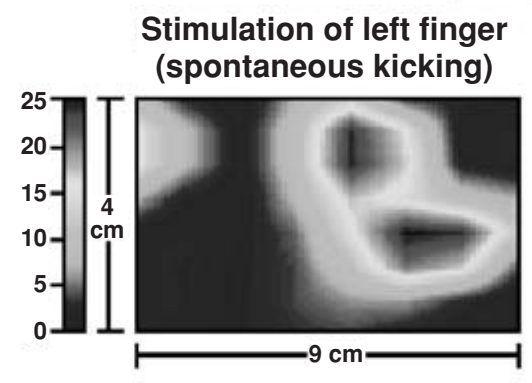

A

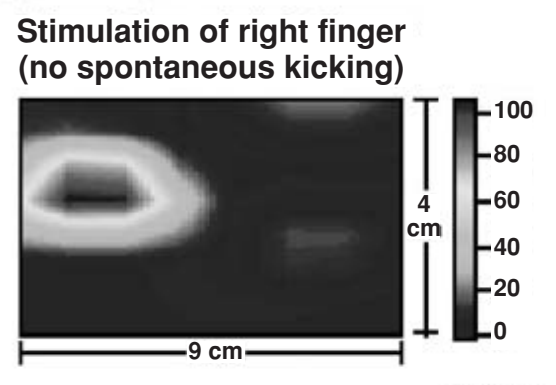

SMZ 101a

B

Figure 13. Somatosensory and motor activation in an infant. Reproduced with permission from (Chance et al., 1998a).

\subsubsection{Optical Tomography of the Brain}

The optical properties of the adult head and its large diameter limit the applicability of tomographic reconstruction methods. The smaller dimensions of the neonatal head together with more transparent optical properties and thinner CSF layer make it an easier problem.

The main clinical interest in optical tomography of the neonatal head is in the possibility of detecting and localising abnormalities in cerebral perfusion. If difference data between a reference phantom and the head at two or more wavelengths are available, it is possible to calculate three-dimensional images of the blood volume and oxygen saturation.

First results of the application of time-resolved optical tomography on a premature neonate are presented in (Hebden et al., 2002). The authors used a custom-made helmet with 31 sources and detectors to record data on a prematurely born neonate with cerebral haemorrhage primarily in the left ventricle. Three-dimensional images of absorption and scattering at two wavelengths were reconstructed based on differences in the mean time data type between a reference medium and the subject. The images reveal high absorption on the left side of the brain, which is consistent with ultrasonic localisation of the haemorrhage. The results are promising in that a model based on the diffusion equation was adequate to produce useful images. Improvements in the images can probably be obtained by more sophisticated modeling of scattering and the application of several data types.

Reconstructions of hemodynamic changes in the adult head based on intensity data have been reported in (Bluestone et al., 2001). The authors repeatably performed the Valsava maneuver on the subject, and reconstructed a time-series of images representing the changes in the concentrations of oxy- and deoxyhemoglobin in a volume under the forehead of the subject.

\section{DISCUSSION}

Optical imaging using diffuse near-infrared light is a promising functional imaging modality. Due to its sensitivity to changes in the blood volume and oxygen saturation, it can be used to study a variety of physiological processes in the brain, the breast and muscle 
tissue. At present, much experimental research in the field is based on relatively simple models, but 3D finite-element numerical models are becoming more widespread.

In its most basic form, optical imaging is used to measure changes in the concentrations of oxy- and deoxyhemoglobin in an area of tissue by comparing the measured intensities of light between two different states of tissue ("active" vs. "rest"). A photon propagation model can be used to calculate the spatial sensitivity profiles for the different optode positions and this can be used to improve the accuracy of the functional maps. If both transmitted and reflected light can be measured from the tissue volume, and if a sufficient number of optodes are available, a tomographic reconstruction of the background optical properties is possible. This will further increase the accuracy of the localisation and quantification of the dynamic changes.

In addition to imaging dynamic processes, it is possible to reconstruct the static optical properties of tissue. The optical properties at several wavelengths can be used to reveal abnormalities such as haemorrhage or a tumor in tissue. Static recon-structions generally require either precise calibration or a reference measurement on a phantom. Both breast cancer and haemorrhage in a premature infant's brain have been successfully imaged using reference phantoms, thus indicating that difference imaging combined with nonlinear iterative inversion algorithms has considerable potential in clinical applications.

The standard modeling technique, numerical solution of the diffusion equation, is under pressure to be improved in several respects. The existence of nonscattering regions in the head calls for the solution of a hybrid model. Anisotropic scattering in the white matter or muscle tissue can be incorporated in form of a modified diffusion equation. The solution of the higher order approximations and RTE itself has also been considered. The disadvantage of these methods is the increase in the number of parameters to be solved in the inverse problem, necessitating the use of prior information. The computational requirements of the more sophisticated models are unfortunately quite high.

Although continuous-wave techniques are widely used, time- and frequency-domain techniques provide additional information which is likely to improve the quality of the images. The separation of scattering and absorption in breast and muscle studies is possible. In brain studies, only one data type has been used in most cases, producing inevitable crosstalk between the two optical properties. Further development of time-resolved instruments include improvements in the speed of data acquisition and the accuracy of measured data through better calibration procedures and improved system stability. Of great practical importance is the improvement of the instrument-patient interface.

In activation studies, the development of event-related protocols may increase the variety of cognitive phenomena that can be observed using optical imaging. It may also prove to be a more robust technique for observing brain activity, since high-pass filtering can be applied to reduce the effect of variable optode-tissue contact. Clinical applications of optical activation imaging are likely to be found for infants. Presently, the most practical instruments for activation studies are based on DC measurements.

In muscle imaging, valuable information of, e.g., local changes in the oxydative metabolism of skeletal muscle, can be obtained using only a few channels. Improvements in quantitative accuracy can be achieved by using multichannel instrumentation together with model-based reconstruction techniques.

In a layered structure, the measured change can be partly due to changes in the properties of other layers (e.g., dermis and adipose tissue) above the layer of interest (skeletal 
muscle). A clinically useful imaging modality requires a higher spatial resolution than what is typically achieved today. Measurements at several source-detector distances and a tomographic reconstruction may allow the differentiation of the contributions from different layers.

Due to the non-invasive and non-ionizing nature of NIR light, diffuse optical imaging may provide new possibilities for mammography. Even continuous or regular monitoring is possible. The relatively small size and low price of the instruments together with the fact that no special operational environment is needed mean that optical mammography could be widely used in the clinical environment. Sensitivity for hemodynamic and oxygenation changes may enable an earlier detection of breast cancer and thereby an earlier start for treatment. This would increase the probability of recovery and reduce the need for the excision of the breast.

Despite the relatively low spatial resolution obtained with present optical mammography systems, preliminary results have already shown that the method can provide unique information for breast-cancer detection.

Research on optical mammography should focus on improving measurement and reconstruction techniques. Further testing is required to demonstrate its ability to detect different types of breast cancer, such as metastasis and lymphoma, and to distinguish cancerous tissues from benign lesions. The research of different endogenous chromophores is important as well. In addition to the changes in total hemoglobin and oxygen saturation, local changes in the concentrations of water and lipids may be of diagnostic interest. The concentration of water may increase (and lipids decrease) at the tumor site, indicating the replacement of the dominant adipose tissue by cancerous tissue containing more blood. Exogeneous contrast agents may further improve the contrast in optical mammography without a serious risk to the patient.

ACKNOWLEDGMENTS. The authors would like to acknowledge all the reserchers that allowed the reproduction of material and results from their research and publications.

This work was financially supported by the Academy of Finland, Jenny and Antti Wihuri Foundation, TES, the Finnish Cultural Foundation, and the graduate school Functional Studies in Medicine.

\section{REFERENCES}

Arridge, S. R., Cope, M., and Delpy, D. T., 1992, The theoretical basis for the determination of optical pathlengths in tissue: temporal and frequency analysis, Phys. Med. Biol. 37:1531-1560.

Arridge, S. R., Schweiger, M., Hiraoka, M., and Delpy, D. T., 1993, A finite element approach for modeling photon transport in tissue, Med. Phys. 20:299-309.

Arridge, S. R., 1995, Photon-measurement density functions. Part 1: Analytical forms, Appl. Opt. 34:7395-7409.

Arridge, S. R., and Schweiger, M., 1995, Photon-measurement density functions. Part 2: Finiteelement-method calculations, Appl. Opt. 34:8026-8037.

Arridge, S. R., and Lionheart, W. R. B., 1998, Non-uniqueness in diffusion-based optical tomography, Opt. Lett. 23:882-884.

Arridge, S. R., and Schweiger, M., 1998, A general framework for iterative reconstruction algorithms in optical tomography, using a finite element method, in: Computational Radiology and Imaging: Therapy and Diagnosis, Borgers, C., and Natterer, F., ed., IMA Volumes in Mathematics and its Applications.

Arridge, S. R., 1999, Optical tomography in medical imaging, Inv. Prob. 15:R41-R93. 
Barbour, R. L., Graber, H. L., Pei, Y., Zhong, S., and Schmitz, C. H., 2001, Optical tomographic imaging of dynamic features of dence-scattering media, J. Opt. Soc. Am. A 18:3018-3036.

Beauvoit, B., and Chance, B., 1998, Time-resolved spectroscopy of mitochondria, cells and tissues under normal and pathological conditions, Mol. Cell. Biochem. 184:445-455.

Bevilacqua, F., Piguet, D., Marquet, P., Gross, J. D., Tromberg, B. J., and Depeursinge, C., 1999, In vivo local determination of tissue optical properties: applications to human brain, Appl. Opt. 38:4939-4950.

Bluestone, A., Abdoulaev, G., Schmitz, C. H., Barbour, R. L., and Hielscher, A. H., 2001, Threedimensional optical tomography of hemodynamics in the human head, Opt. Expr. 9:272-286.

Boas, D., 1996, Diffuse Photon Probes of Structural and Dynamical Properties of Turbid Media: Theory and Biomedical Applications, Ph.D. thesis, Univ. of Pennsylvania.

Boas, D., O’Leary, M. A., Chance, B., Yodh, A. G., 1997, Detection and characterization of optical inhomogeneities with diffuse photon density waves: a signal-to-noise analysis, Appl. Opt. 36:75-92.

Boas, D. A., Gaudette, T., and Arridge, S. R., 2001a, Simultaneous imaging and optode calibration with diffuse optical tomography, Opt. Expr. 8:263-270.

Boas, D. A., Gaudette, T., Strangman G., Cheng, X., Marota, J. J. A., and Mandeville, J. B., 2001b, The accuracy of near infrared spectroscopy and imaging during focal changes in cerebral hemodynamics, Neurolmage 13:76-90.

Boas, G., 2002, Optical Breast Imaging: Mammography’s next step? Advance for Imaging and Radiation Therapy Professionals June 17:30-31.

Bohren, C. F., and Huffman, D. R., 1983, Absorption and Scattering of Light by Small Particles, J. Wiley, New York.

Bolin, F. P., Preuss, L. E., Taylor, R. C., and Ference, J., 1989, Refractive index of some mammalian tissues using a fiber optic cladding method, Appl. Opt. 28:2297-2296.

Boushel, R., and Piantadosi, C. A., 2000, Near-infrared spectroscopy for monitoring muscle oxygenation, Acta Physiol. Scand. 168:615-622.

Case, K. M., and Zweifel, P. F., 1967, Linear Transport Theory, Addison-Wesley.

Cerussi, A. E., Jakubowski, D., Shah, N., Bevilacqua, F., Lanning, R., Berger, A. J., Hsiang D., Butler J., Holcombe R. F., and Tromberg B. J., 2002, Spectroscopy enhances the information content of optical mammography, J. Biomed. Opt. 7:60-71.

Chance, B., Leigh, J. S., Miyake, H., Smith, D. S., Nioka, S., Greenfeld, R., Finander, M., Kaufmann, K., Levy, W., Young, M., Cohen, P., Yoshioka, H., and Boretsky, R., 1988, Comparison of time resolved and unresolved measurements of deoxyhemoglobin in brain. Proc. Nat. Acad. Sci. USA 85:4971-4975.

Chance, B., Zhuang, Z., UnAh, C., Alter, C., and Lipton, L., 1993, Cognition-activated low-frequency modulation of light absorption in human brain, Proc. Natl. Acad. Sci. USA 90:3770-3774.

Chance, B., Anday, E., Nioka, S., Zhou, S., Hong, L., Worden, K., Li, C., Murray, T., Ovetsky, Y., Pidikiti, D., and Thomas, R., 1998a, A novel method for fast imaging of brain function, noninvasively, with light. Opt. Expr. 2:411-423.

Chance, B., Cope, M., Gratton, E., Ramanujam, N., and Tromberg, B., 1998b, Phase measurement of light absorption and scatter in human tissue, Rev. Sci. Instrum. 69:3457-3481.

Chandrasekhar, S., 1960, Radiative. Transfer, Dover Publications, Inc., New York.

Chen, Y., Zhou, S., Xie, C., Nioka, S., Delivoria-Papadopoulos, M., Anday, E., and Chance, B., 2000, Preliminary evaluation of dual wavelength phased array imaging on neonatal brain function, J. Biomed. Opt. 5:194-200.

Cheong, W., Prahl, S. A., and Welch, A. J., 1990, A review of the optical properties of biological tissues, IEEE J. Quant. Elec. 26:2166-2185.

Colak, S. B., van der Mark, M. B., Hooft, G. W.'t, Hoogenraad, J. H., van der Linden, E. S., and Kuijpers, F. A., 1999, Clinical optical tomography and NIR spectroscopy for Breast Cancer Detection, IEEE J. Quantum Electron. 5:1143-1158.

Cooper, C. E., Matcher, S. J., Wyatt, J. S., Cope, M., Brown, G. C., Nemoto, E. M., and Delpy, D. T., 1994, Near infrared spectroscopy of the brain: relevance to cytochrome oxidase bioenergetics, Biochem. Soc. Trans. 22:974-980.

Cooper, C. E., and Springett, R., 1997, Measurement of cytochrome oxidase and mitochondrial energetics by near-infrared spectroscopy, Phil. Trans. R. Soc. London Ser. B 352:669-677.

Cope, M., 1991, The Development of a Near Infrared Spectoscopy System and its Application for Non Invasive Monitoring of Cerebral Blood and Tissue Oxygenation in the Newborn Infant, Ph.D. thesis, University of London. 
Culver, J. P., Siegel, A., Franceschini, M. A., Marota, J. J., Mandeville, J. B., and Boas, D. A., 2002, OSA Biomedical Topical Meetings, OSA Technical Digest, (Optical Society of America, Washington DC), pp. 4143.

Colier, W. N., Quaresima, V., Wenzel, R., van der Sluijs, M. C., Oeseburg, B., Ferrari, M., and Villringer, A., 2001, Simultaneous near-infrared spectroscopy monitoring of left and right occipital areas reveals contra-lateral hemodynamic changes upon hemi-field paradigm, Vision Res. 41:97-102.

De Blasi, R. A., Fantini, S., Franceschini, M. A., Ferrari M., and Gratton, E., 1995, Cerebral and muscle oxygen saturation measurement by a novel frequency-domain near-infrared spectrometer, Med. \& Biol. Eng. \& Comput. 33:228-230.

Den Outer, P. N., Nieuwenhuizen, T. M., and Lagendijk, A., 1993, Location of objects in multiple-scattering media, J. Opt. Soc. Am. A 10:1209-1218.

Dehghani, H., Pogue, B. W., Poplack, S. P., and Paulsen, K. D., 2003, Multiwavelength threedimensional nearinfrared tomography of the breast: initial simulation, phantom, and clinical results, Appl. Opt. 42:135-145.

Delpy, D. T., Cope, M., van der Zee, P., Arridge, S., Wray, S., and Wyatt, J., 1988, Estimation of optical pathlength through tissue from direct time of flight measurement, Phys. Med. Biol. 33:1433-1442.

DiCon FiberOptics, 1998, VX500 fiberoptic switch operation manual, Berkeley, USA.

Dorn, O., 1998, A transport-backtransport method for optical tomography, Inv. Prob. 14:1107-1130.

Dorn, O., 2000, Scattering and absorption transport sensitivity functions for optical tomography, Optics Express 7:492-506.

Dorn, O., submitted, Shape reconstruction in scattering media with voids using a transport model and level sets, Canadian Applied Mathematics Quarterly

Duncan, A., Meek, J. H., Clemence, M., Elwell, C. E., Tyszczuk, L., Cope, M., and Delpy, D., 1995, Optical pathlength measurements on adult head, calf and forearm and the head of the newborn infant using phase resolved optical spectroscopy, Phys. Med. Biol. 40:295-304.

Durduran, T., Choe, R., Culver, J. P., Zubkov, L., Holboke, M. J., Chance, B., and Yodh, A. G., 2002, Bulk optical properties of healthy female breast tissue, Phys. Med. Biol. 47:2847-2861.

Ebert B., Sukowski U., Grosenick D., Wabnitz H., Moesta T. K., Licha K., Becker A., Semmler W., Schlag P. M., and Rinneberg H., 2001, Near-infrared fluorescent dyes for enhanced contrast in optical mammography: phantom experiments, J. Biomed. Opt. 6:134-140.

Eda, H., Oda, Ichiro, Yasunobu, I., Wada, Y., Oikawa, Y. Tsunazawa, Y., Takada, M., Tsuchiya, Y., Yamashita, Y., Oda, M., Sassaroli, A., Yamada, Y., and Tamura, M., 1999, Multichannel time-resolved optical tomographic imaging system, Rev. Sci. Instrum. 70:3595-3601.

Elwell, C. E., 1995, A practical users guide to near infrared spectroscopy, UCL Reprographics, Lontoo.

Elwell, C. E., Owen-Reece, H., Wyatt, J. S., Cope, M., Reynolds, E. O., and Delpy, D. T., 1996, Influence of respiration and changes in expiratory pressure on cerebral haemoglobin concentration measured by near infrared spectroscopy, J. Cereb. Blood Flow Metab. 16:353-357.

Fantini, S. and Franceschini, M. A., 2002, Frequency-domain techniques for tissue spectroscopy and imaging, in: Handbook of Optical Biomedical Diagnostics, V. V. Tuchin, ed., SPIE Press, Belllingham, Washington, pp. 405-453.

Fantini, S., Walker, S. A., Franceschini, M. A., Kaschke, M., Schlag, P. M., and Moesta, K. T., 1998, Assessment of the size, position, and optical properties of breast tumors in vivo by noninvasive optical methods, Appl. Opt. 37:1982-1989.

Feng, S., Zeng, F., and Chance, B., 1995, Photon migration in the presence of a single defect: a perturbation analysis, Appl. Opt. 34:3826-3837.

Ferrari, M., Binzoni, T., and Quaresima, V., 1997, Oxidative metabolism in muscle, Phil. Trans. R. Soc. Lond. B 352:677-683.

Firbank, M., and Delpy, D. T., 1993, A design for a stable and reproducible phantom for use in near-infrared imaging and spectroscopy, Phys. Med. Biol. 38:847-853.

Firbank, M., Oda, M., and Delpy, D. T., 1995, An improved design for a stable and reproducible phantom material for use in near-infrared spectroscopy and imaging, Phys. Med. Biol. 40:955-961.

Firbank, M., Hiraoka, M., Essenpreis, M., and Delpy, D. T., 1997, Measurement of the optical properties of the skull in the wavelength range 650-950 nm, Phys. Med. Biol. 38, 503-510.

Fishkin, J. B., and Gratton, E., 1993, Propagation of photon-density waves in strongly scattering media containing an absorbing semi-infinite plane bounded by a straight edge, J. Opt. Soc. Am. A 10:127-140. 
Fishkin, J. B., Coquoz, O., Anderson, E. R., Brenner, M., and Tromberg, B. J., 1997, Frequency-domain photon migration measurements of normal and malignant tissue optical properties in a human subject, Appl. Opt. 36:10-20.

Franceschini, M. A., Moesta, K. T., Fantini, S., Gaida, G., Gratton, E., Jess, H., Mantulin, W. W., Seeber, M., Schlag, P. M., and Kaschke, M., 1997, Frequency-domain techniques enhance optical mammography: initial clinical results, Proc. Natl. Acad. Sci. USA 94:6468-6473.

Franceschini, M. A., Gratton, E., and Fantini, S., 1999, Non-invasive optical method to measure tissue and arterial saturation: an application to absolute pulse oximetry of the brain, Opt. Lett. 24:829-831.

Franceschini, M. A., Toronov, V., Filiaci, M. E., Gratton, E., and Fantini, S., 2000, On-Line Optical Imaging of the Human Brain with 160 ms Temporal Resolution, Opt. Expr. 6:49-57.

Frostig, R. D., Lieke, E. E., Ts'o, D. Y., and Grinvald, A., 1990, Cortical functional architecture and local coupling between neuronal activity and the microcirculation revealed by in vivo high-resolution optical imaging of intrinsic signals, Proc. Natl. Acad. Sci. USA 87:6082-6086.

Gibbs, E. L., Lennox, W. G., Nims, L. F., and Gibbs, F. A., 1942, Arterial and cerebral venous blood, arterial-venous differences in man, J. Biol. Chem. 144:325-332.

Graaff, R., Aarnoudse, J. G., de Mul, F. F. M., and Jentink, H. W., 1989, Light propagation parameters for anisotropically scattering media based on a rigorous solution of the transport equation, Appl. Opt. 28:22732279.

Gratton, G., and Fabiani, M., 2001, The event-related optical signal: a new tool for studying brain function, Int. J. of Psychophys. 42:109-121.

Groenhuis, R. A. J., Ferwerda, H. A., and Ten Bosch, J. J., 1983a, Scattering and absorption of turbid materials determined from reflection measurements 1: theory, Appl. Opt. 22:2456-2462.

Groenhuis, R. A. J., Ten Bosch, J. J., and Ferwerda, H. A., 1983b, Scattering and absorption of turbid materials determined from reflection measurements 2: measuring method and calibration, Appl. Opt. 22:24632467.

Grosenick, D., Wabnitz, H., Rinneberg, H., Moesta, K. T., and Schlag, P., 1999, Development of a time-domain optical mammograph and first in vivo applications, Appl. Opt. 38:2927-2943.

Grosenick, D., Wabnitz, H., Macdonald, R., Rinneberg, H., Mucke, J., Stroszczynski, C., Moesta, K. T., and Schlag, P., 2002, Determination of in vivo optical properties of breast tissue and tumors using a laser pulse mammography, in: OSA Biomedical Topical Meetings, OSA Technical Digest, Optical Society of America, Miami Beach, Florida, pp. 459-461.

Haskell, R. C., Svaasand, L. O., Tsay, T-T., Feng, T-C., McAdams, M. S., and Tromberg, B. J., 1994, Boundary conditions for the diffusion equation in radiative transfer, J. Opt. Soc. Am. A 11:2727-2741.

Hargrave, P., Nicholson, P. W., Delpy, D. T., and Firbank, M., 1996, Optical properties of multicellular tumor spheroids, Phys. Med. Biol. 41:1067-1072.

Hawrysz, D. J., and Sevick-Muraca, E. M., 2000, Developments toward diagnostic breast cancer imaging using near-infrared optical measurements and fluorescent contrast agents, Neoplasia 2:388-417.

Hebden, J. C., Gibson, A., Yusof, R. M., Everdell, N., Hillman, E. M. C., Delpy, D. T., Arridge, S. R., Austin, T., Meek, J. H., and Wyatt, J. S., 2002a, Three-dimensional optical tomography of the premature infant brain, Phys. Med. Biol. 47:4155-4166.

Hebden, J. C., Gonzalez, F. M., Gibson, A., Hillman, E. M. C., Yusof, R., Everdell, N., Delpy, D. T., Zaccanti, G., and Martelli, F., 2002b, Assessment of an in situ temporal calibration method for time-resolved optical tomography, J. Biomed. Opt. (In press).

Heino, J. and Somersalo, E., 2002, Estimation of optical absorption in anisotropic background, Inv Prob. 18:559573.

Hielscher, A. H., Alcouffe, R. E., Barbour, R. L., 1997, Transport and diffusion calculations on MRI generated data, in: Optical Tomography and Spectroscopy of Tissue: Theory, Instrumentation, Model, and Human Studies, B. Chance, and R.R. Alfano, ed., Proc. SPIE 2979, pp. 500-508.

Hielscher, A. H., Klose, A. D., and Hanson, K. M., 1999, Gradient-based iterative image reconstruction scheme for time-resolved optical tomography, IEEE Transactions on Medical Imaging 18:262-271.

Hill, D. K., and Keynes, R. D., 1949, Opacity changes in stimulated nerve, J. Physiol. 108:278-281.

Hillman, E. M. C., Hebden, J. C., Schmidt, F. E. W., Arridge, S. R., Schweiger, M., Dehghani, H., and Delpy, D. T., 2000, Calibration techniques and datatype extraction for time-resolved optical tomography, Rev. Sci. Instrum. 71:3415-3427. 
Hillman, E. M. C., Hebden, J. C., Schweiger, M., Dehghani, H., Schmidt, F. E. W., Delpy, D. T., and Arridge, S. R., 2001, Time resolved optical tomography of the human forearm, Phys. Med. Biol. 46: 1117-1130.

Hillman, E. M. C., 2002, Experimental and Theoretical Investigations of Near Infrared Tomographic Imaging Methods and Clinical Applications, Ph.D. thesis, University of London.

Hintz, S. R., Benaron, D. A., Siegel, A. M., Zourabian, A., Stevenson, D. K., and Boas, D. A., 2001, Bedside functional imaging of the premature infant brain during passive motor activation, J. Perinat. Med. 29:335343.

Holboke, M. J., Tromberg, B. J., Li, X., Shah, N., Fishkin, J., Kidney, D., Butler, J., Chance, B., and Yodh, A. G., 2000, Three-dimensional diffuse optical mammography with ultrasound localization in a human subject, $J$. Biomed. Opt. 5:237-247.

Hoshi, Y. and Tamura, M., 1993, Detection of dynamic changes in cerebral oxygenation coupled to neuronal function during mental work in man, Newrosci. Lett. 150:5-8.

Ishimaru, A., 1978, Wave Propagation and Scattering in Random Media, Vol. 1, Single Scattering and Transport Theory, Academic Press.

Israel, H., Obrig, H., Kohl, M., Uludag, K., Müller, B., Wenzel, R., Buckow, C., Arnold, G., Villringer, A., 2001, Is neurovascular coupling altered in interictal migraineurs? A combined visually evoked potential (VEP) and near infrared spectroscopy (NIRS) approach, Neurolmage 13:S800.

Iwata N. K., Ugawa, Y., Watanabe, E., Yamashita, Y., Koizumi, H., and Kanazawa, I., 2000, Interhemispheric connection between bilateral hand motor areas studied with near-infrared spectroscipic (NIRS) mapping and transcramal magnetic stimulation (TMS), Neurolmage 11:S520.

Jiang, H., 1999, Optical image reconstruction based on the third-order diffusion equations, Optics Express 4:241246.

Jiang, H., Xu, Y., Iftimia, N., Eggert, J., Klove, K., Baron, L., and Fajardo, L., 2001, Three-dimensional optical tomographic imaging of breast in a human subject, IEEE Trans. Med. Imaging 20:13341340 .

JöUbsis, F. F., 1977, Noninvasive, infrared monitoring of cerebral and myocardial oxygen sufficiency and circulatory parameters, Science 198:1264-1267.

Kaipio, J. P., Kolehmainen, V., Somersalo, E., and Vauhkonen, M., 2000, Statistical inversion and Monte Carlo sampling methods in electrical impedance tomography, Inv. Prob. 16:1487-1522.

Kennan, R. P., Horovitz, S. G., Maki, A., Yamashita, Y., Koizumi, H., Gore, J. C., 2002, Simultaneous Recording of Event-Related Auditory Oddball Response Using Transcranial Near Infrared Optical Topography and Surface EEG. Neurolmage 16:587-592.

Kim. A. D. and Ishimaru, A., 1998 Optical diffusion of continuous-wave, pulsed, and density waves in scattering media and comparisons with radiative transfer, Appl. Opt. 37:5313-5319.

Kleinschmidt, A., Obrig, H., Requardt, M., Merboldt, K. D., Dirnagl, U., Villringer, A., Frahm, J., 1996, Simultaneous recording of cerebral oxygenation changes during human brain activation by magnetic resoncance imaging and near - infrared spectroscopy, J. Cereb. Blood Flow Metab. 16:817-827.

Klose, A. D., Netz, U., Beuthan, J., and Hielscher, A. H., 2002, Optical tomography using the time-independent equation of radiative transfer - Part 1: forward model, Journal of Quantitative Spectroscopy \& Radiative Transfer 72:691-713.

Klose, A. D. and Hielscher, A. H., 2002, Optical tomography using the time-independent equation of radiative transfer - Part 2: inverse model, Journal of Quantitative Spectroscopy \& Radiative Transfer 72:715732.

Kohl-Bareis, M., Buckow, C., Zank, H., Obrig, H., Steinbrink, J., and Villringer, A., 2002, Multi channel NIR topography for the assessment of cortical activation, OSA Biomedical Topical Meetings, OSA Technical Digest, (Optical Society of America, Washington DC), pp. 300-303.

Kolehmainen, V., 2001, Novel Approaches to Image Reconstruction in Diffusion Tomography, PhD thesis, University of Kuopio.

Kusaka, T., Isobe, K., Nagano, K., Okubo, K., Yasuda, S., Kondo, M., Itoh, S., and Onishi, S., 2001, Neurolmage 13:944-952.

Laufer, J., Simpson, C. R., Kohl, M., Essenpreis, M., and Cope, M., 1998, Effect of temperature on the optical properties of ex-vivo human dermis, Phys. Med. Biol. 43:2479-2490.

Lovell, A. T., Hebden, J. C., Goldstone, J. C., and Cope, M., 1999a, Determination of the transport scatter coefficient of red blood cells, Proc. SPIE 3597:175-182. 
Lovell, A. T., Owen-Reece, H., Elwell, C. E., Smith, M., and Goldstone J. C., 1999b, Continuous measurement of cerebral oxygenation by near infrared spectroscopy during induction of anesthesia, Anaesthesiology and Analgesia 88, 554-558.

Mackert, B. M., Wübbeler, G., Leistner, S., Burghoff, M., Uludag, K., Obrig, H., Kohl, M., and Villringer, A., 2002, Proceedings of the 13th International Conference on Biomagnetism, August 10-14, Jena, Germany, p. 850.

MacVicar, B. A. and Hochman, D., 1991, Imaging of synaptically evoked intrinsic optical signals in hippocampal slices, J. Neurosci. 11:1458-1469.

Maris, M., Gratton, E., Maier, J., Mantulin W., and Chance, B., 1994, Functional near-infrared imaging of deoxygeneted haemoglobin during exercise of the finger extensor muscles using the frequency-domain technique, Bioimaging 2:174-183.

Markel, V. A. and Schotland, J. C., 2001, Inverse problem in optical diffusion tomography. I. Fourier-Laplace inversion formulas, J. Opt. Soc. Am. A 18:1336-1347.

Marquez, G., Wang, L. V., Lin, S. P., Schwartz, J. A., and Thomsen, S. L., 1998, Anisotropy in the absorption and scattering spectra of chicken breast, Appl. Opt. 37:798-805.

Matcher, S. J., Cope, M., and Delpy, D. T., 1994, Use of the water absorption spectrum to quantify tissue chromophore concentration changes in near infrared spectroscopy, Phys. Med. Biol. 39:177-196.

Matcher, S. J., 1999, Nonuniqueness in optical tomography: relevance of the P1 approximation, Opt. Lett. 24:1729_ 1731.

Matson, C. L. and Liu, H., 1999, Backpropagation in turbid media, J. Opt. Soc. Am. A 16:1254-1265.

McBride, T. O., Pogue, B. W., Jiang, S., Österberg, U. L., and Paulsen, K. D., 2001, A parallel-detection frequencydomain near-infrared tomography system for hemoglobin imaging of the breast in vivo, Rev. Sci. Instrum. 72:1817-1824.

Moesta, K. T., 2002, Clinical evaluation of optical breast imaging: what requirements of the clinician can be fulfilled, in: OSA Biomedical Topical Meetings, OSA Technical Digest, Optical Society of America, Miami Beach, Florida, pp. 432-434.

Mourant, J. R., Freyer, J. P., Hielscher, A. H., Eick, A. A., Shen, D., and Jonhson, T. M., 1998, Mechanisms of light scattering from biological cells relevant to noninvasive optical-tissue diagnostics, Appl. Opt. 37:3586-3593.

Nioka, S., Miura, H., Long, H., Perry, A., Moser, D., and Chance, B., 1999, Functional muscle imaging in elite and untrained subjects, Proc. SPIE 3597:282-290.

Niwayama, M., Yamamoto, K., Kohota, D., Hirai, K., Kudo, N., Hamaoka, T., Kime, R., and Katsumura, T., 2002, A 200-channel imaging system of muscle oxygenation using CW near-infrared spectroscopy, IEICE Trans. Inf. \& Syst. 85-D:115-123.

Nissilä, I., Kotilahti, K., Fallström, K., and Katila, T., 2002a, Instrumentation for the accurate measurement of phase and amplitude in optical tomography, Rev. Sci. Instrum. 73:3306-3312.

Nissilä, I., Kotilahti, K., Komssi, S., Kähkönen, S., Noponen, T., Ilmoniemi, R.J., and Katila, T., 2002b, Optical measurement of hemodynamic changes in the contralateral motor cortex induced by transcranial magnetic stimulation, Proceedings of the 13th International Conference on Biomagnetism, August 10-14, Jena, Germany, pp. 851-854.

Ntziachristos, V., Ma, X., and Chance, B., 1998, Time-correlated single photon counting imager for simultaneous magnetic resonance and near-infrared mammography, Rev. Sci. Instrum. 69:4221-4233.

Ntziachristos, V., Chance, B., and Yodh, A. G., 1999, Differential diffuse optical tomography, Optics Express 5:230-242.

Ntziachristos, V., Yodh, A. G., Schnall, M., and Chance, B., 2000, Concurrent MRI and diffuse optical tomography of breast after indocyanine green enhancement, Proc. Nat. Acad. Sci. USA 97:2767-2772.

Ntziachristos, V., Yodh, A. G., Schnall, M. D., and Chance, B., 2002, MRI-guided diffuse optical spectroscopy of malignant and benign breast lesions, Neoplasia 4:347-354.

Obrig, H. and Villringer, A., 1997, Near-infrared spectroscopy in functional activation studies: can NIRS demonstrate cortical activation? Adv. Exp. Med. Biol. 413:113-127.

Obrig, H., Neufang, M., Wenzel, R., Kohl, M., Steinbrink, J., Einhäupl, K. M., and Villringer, A., 2000, Spontaneous low frequency oscillations of cerebral hemodynamics and metabolism in human adults, Neurolmage 12:623639.

Obrig, H., Israel, H., Kohl-Bareis, M., Uludag, K., Wenzel, R., Muller, B., Arnold, G., and Villringer A., 2002, Habituation of the visually evoked potential and its vascular response: Implications for neurovascular coupling in the healthy adult, NeuroImage 17:1-18. 
Okada, E., Firbank, M., Schweiger, M., Arridge, S. R., Cope, M., and Delpy, D. T., 1997, Theoretical and experimental investigation of near-infrared light propagation in a model of the adult head, Appl. Opt. 36:2131.

Okada, E., Schweiger, M., Arridge, S. R., Firbank, M., and Delpy, D. T., 1996, Experimental validation of Monte Carlo and finite-element methods for the estimation of the optical path length in inhomogencous tissue, Appl. Opt. 35:3362-3371.

O'Leary, M. A., Boas, D. A., Chance, B., and Yodh, A. G., 1995, Experimental images of heterogeneous turbid media by frequency-domain diffusing-photon tomography, Opt. Lett. 20:426-428.

O'Leary, M. A., Boas, D. A., Chance, B., and Yodh, A. G., 1992, Refraction of diffuse photon density waves, Phys. Rev. Lett. 69:2658-2661.

Oliviero, A., Di Lazzaro, V., Piazza, O., Profice, P., Pennisi, M. A., Della Corte, F., Tonali, P., 1999, Cerebral blood flow and metabolic changes produced by repetitive magnetic brain stimulation, J. Neurol. 246:1164-1168.

Ostermeyer, M. R. and Jacques, S. L., 1997, Perturbation theory for diffuse light transport in complex biological tissues, J. Opt. Soc. Am. A 14:255-261.

Pickering, J. W., Prahl, S. A., van Wieringen, N., Beek, J. F., Sterenborg, H. J. C. M. and van Gemert, M. J. C., 1993, Double-integrating-sphere system for measuring the optical properties of tissue, Appl. Opt. 32: 399.

Poe, G. R., Nitz, D. A., Rector, D. M., Kristensen, M. P., and Harper, R. M., 1996, Concurrent reflectance imaging and microdialysis in the freely behaving cat, J. Neurosci. Methods 65:143-149.

Pham, T. H., Coquoz, O., Fishkin, J. B., Anderson, E., and Tromberg, B. J., 2000, Broad bandwidth frequency domain instrument for quantitative tissue optical spectroscopy, Rev. Sci. Instrum. 71:2500-2513.

Pogue, B. W., Patterson, M. S., Jiang, H., and Paulsen, K. D., 1995, Initial assessment of a simple system for frequency domain diffuse optical tomography, Phys. Med. Biol. 40:1709-1729.

Pogue, B. W., Testorf, M., McBride, T., Osterberg, U., and Paulsen, K. D., 1997, Instrumentation and design of a frequency-domain diffuse optical tomography imager for breast cancer detection, Opt. Expr. 1:391-403.

Pogue, B. W., Poplack, S., Mcbride, T., Wells, W., Osterman, K., Osterberg, U. L., and Paulsen, K. D., 2001, Quantitative hemoglobin tomography with diffuse near-infrared light: pilot results in the breast, Radiology 218:261-266.

Prahl, S. A., Keijzer, M., Jacues, S. L., and Welch, A. J., 1989, A Monte Carlo Model of Light Propagation in Tissue, SPIE Institute series 5:102-111.

Prahl, S. A., van Gemert, M. J. C., and Welch, A. J., 1993, Determining the optical properties of turbid media by using the adding-doubling method, Appl. Opt. 32:559-568.

Quaresima, V., Matcher, S. J., and Ferrari, M., 1998, Identification and Quantification of Intrinsic Optical Contrast for Near-Infrared Mammography, Photochem. Photobiol. 67:4-14.

Quaresima, V., Colier, W. N. J. M., van der Sluijs, M., and Ferrari, M., 2001, Nonuniform quadriceps $\mathrm{O}_{2}$ consumption revealed by near infrared multipoint measurements, Biochem. \& Biophys. Res. Comm. 285:1034-1039.

Rector, D. M., Rogers, R. F., Schwaber, J. S., Harper, R. M., and George, J. S., 2001, Scattered-light imaging in vivo tracks fast and slow processes of neurophysiological activation, NeuroImage 14:977-994.

Rinzema, K., Murrer, L. P. H., and Star, W. M., 1998, Direct experimental verification of light transport theory in an optical phantom, J. Opt. Soc. Am. A 15:2078-2088.

Sako, T., Hamaoka, T., Higuchi, H., Kurosawa, Y., and Katsumura, T., 2001, Validity of NIR spectroscopy for quantitatively measuring muscle oxidative metabolic rate in exercise, J. Appl. Physiol. 90:338-344.

Sato, H., Takeuchi, T., and Sakai, K. L., 1999, Temporal cortex activation during speech recognition: an optical topography study, Cognition 73:B55-66.

Schmidt, F. E. W., Fry, M. E., Hillman, E. M. C., Hebden, J. C., and Delpy, D. T., 2000, A 32-channel time-resolved instrument for medical optical tomography, Rev. Sci. Instrum. 71:256-265.

Schmitz, C. H., Graber, H. L., Luo, H., Arif, I., Hira, J., Pei, Y., Bluestone, A., Zhong, S., Andronica, R., Soller, I., Ramirez, N., Barbour, S.-L.S., and Barbour, R. L., 2000, Instrumentation and calibration protocol for imaging dynamic features in dense-scattering media by optical tomography, Appl. Opt. 39: 6466-6486.

Schmitz, C. H., Löcker, M., Lasker, J. M., Hielscher, A. H., and Barbour, R. L., 2002, Instrumentation for fast functional optical tomography, Rev. Sci. Instrum. 73:429-439.

Schotland, J. C., Haselgrove, J. C., and Leigh, J. S., 1993, Photon hitting density, Appl. Opt. 32:448-453.

Schweiger, M., Arridge, S. R., Hiraoka, M., and Delpy, D. T., 1995, The finite element method for the propagation of light in scattering media: Boundary and source conditions, Med. Phys. 22:1779-1792. 
Schweiger, M. and Arridge, S. R., 1997, Optimal data types in optical tomography, Information Processing in Medical Imaging (IPMI'97 Proceedings) (Lecture notes in Computer Science 1230), pp. 71-84.

Schweiger, M. and Arridge, S. R., 1999a, Application of temporal filters to time resolved data in optical tomography, Phys. Med. Biol. 44:1699-1717.

Schweiger, M. and Arridge, S. R., 1999b, Optical tomographic reconstruction in a complex head model using a priori region boundary information, Phys. Med. Biol. 44:2703-2722.

Simpson, C. R., Kohl, M., Essenpreis, M., and Cope, M., 1998, Near infrared optical properties of exvivo human skin and sub-cutaneous tissues measured using the Monte Carlo inversion technique, Phys. Med. Biol. 43:2465-2478.

Steinbrink, J., Kohl, M., Obrig, H., Curio, G., Syre, F., Thomas, F., Wabnitz, H., Rinneberg, H., Villringer, A., 2000, Somatosensory evoked fast optical intensity changes detected non-invasively in the adult human head, Neurosci. Lett. 291:105-108.

Steinbrink, J., Wabnitz, H., Obrig, H., Villringer, A., Rinneberg, H., 2001, Determining changes in NIR absorption using a layered model of the human head, Phys. Med. Biol. 46:879-96.

Stepnoski, R. A., LaPorta, A., Raccuia-Behling, F., Blonder, G. E., Slusher, R. E., and Kleinfeld, D., 1991, Noninvasive detection of changes in membrane potential in cultured neurons by light scattering, Proc. Natl. Acad. Sci. USA 88:9382-9386.

Toronov, V., Webb, A., Choi, J. H., Wolf, M., Safonova, L., Wolf, U., and Gratton, E., 2001, Opt. Expr. 9:417-427.

Tromberg, B. J., Coquoz, O., Fishkin, J. B., Pham, T., Anderson, E., Butler, J., Cahn, M., Gross, J. D., Venugopalan, V., and Pham, D., 1997, Non-invasive measurements of breast tissue optical properties using frequency domain photon migration, Phil. Trans. R. Soc. London Ser. B 352:661-668.

Tromberg, B. J., Shah, N., Lanning, R., Cerussi, A., Espinoza, J., Pham, T., Svaasand, L., Butler, J., 2000, Noninvasive in vivo characterization of breast tumors using photon migration spectroscopy, Neoplasia 2:1-15.

Troy, T. L., Page, D. L., and Sevick-Muraca, E. M., 1996, Optical properties of normal and diseased breast tissues: prognosis for optical mammography, J. Biomed. Opt. 1:342-355.

Tuchin, V. V., 2000, Light scattering methods and instruments for medical diagnosis, Tutorial texts series TT38, SPIE, Bellingham, Washington.

University College London, 2002, Biomedical Optics Research Group. Muscle Imaging (London, December, 2002); http://www.medphys.ucl.ac.uk/research/borg/research/monstir/muscle.htm.

Vaithianathan, T., Tullis, I., Meek, J., Austin, T., Delpy, D., 2002, A portable near infrared spectroscopy system for mapping functional activation on infants, 8th International Conference on Functional Mapping of the Human Brain.

Van Beekvelt, M. C. P., Borghuis, M. S., van Engelen, B. G. M., Wevers R. A., and Colier W. N. J. M., 2001, Adipose tissue thickness affects in vivo quantitative near-IR spectroscopy in human skeletal muscle, Clin. Sci. 101:21-28.

Van der Zee, P., 1992, Measurement and modeling of the optical properties of biological tissues in the near infrared, Ph.D., University of London.

Van der Zee, P., Essenpreis, M., and Delpy, D. T., 1993, Optical properties of brain tissue, Proc. SPIE 1888:454465.

Villringer, A. and Dirnagl, U., 1995, Coupling of brain activity and cerebral blood flow: basis of functional neuroimaging, Cerebrovasc. Brain Metab. Rev. 7:240-276.

Villringer, A., 1997, Functional neuroimaging: optical approach, Adv. Exp. Med. Biol. 413:1-18.

Villringer, A. and Chance, B., 1997, Non-invasive optical spectroscopy and imaging of human brain function, Trends Neurosci. 20:435-442.

Walker, S. C., Fentini, S., and Gratton, E., 1997, Image reconstruction by backprojection from frequency-domain optical measurements in highly scattering media, Appl. Opt. 36:170-179.

Wang, L. and Jacques, S. L., 1993, Hybrid model of Monte Carlo simulation and diffusion theory for light reflectance by turbid media, J. Opt. Soc. Am. A 10:1746-1752.

Wilson, B. C. and Adam, G., 1983, A Monte Carlo model for the absorption and flux distributions of light in tissue, Med. Phys. 10:824-830.

Wilson, B. C., Patterson, M. S., and Flock, S. T., 1987, Indirect versus direct technologies for the measurment of the optical properties of tissues, Photochem. Photobiol. 36:601-608.

Woodard, H. Q., and White, D. R., 1986, The composition of body tissues, Br. J. Radiol. 59:1209-1219.

Zhang, Q., Ma, H., Nioka, S., and Chance, B., 2000, Study of near infrared technology for intracranial hematoma detection, J. Biomed. Opt. 5:206-213. 